\title{
Magnetosonic resonances in the magnetospheric plasma
}

\author{
A. S. Leonovich and D. A. Kozlov \\ Institute of Solar-Terrestrial Physics of the Siberian Branch of the Russian Academy of Science, Irkutsk, Russia
}

(Received February 10, 2012; Revised June 9, 2012; Accepted July 7, 2012; Online published June 10, 2013)

\begin{abstract}
A problem of coupling between fast and slow magnetosonic waves in Earth's magnetosphere (magnetosonic resonance) is examined. Propagation both slow magnetosonic wave and Alfven wave can easily be canalized along the magnetic field line direction. The main difference between the two is that slow magnetosonic waves dissipate strongly due to their interaction with the background plasma ions, whose temperature is above the electron temperature. In Earth's magnetosphere, however, there is a region where the dissipation of slow magnetosonic waves can be weak - the inner plasmasphere. The slow magnetosonic waves generated there can be registered directly. In other regions, with strong dissipation of slow magnetosonic waves, their signature may be detected through their impact on the Alfven resonance at frequencies for which the resonant Alfven and slow magnetosonic waves exist simultaneously in the magnetosphere. Owing to their strong coupling with the background plasma ions, resonant slow magnetosonic waves can transfer the energy and impulse from the solar wind to the magnetospheric plasma ions via fast magnetosonic waves penetrating into the tail lobes. A problem of resonant conversion of fast magnetosonic waves into slow magnetosonic oscillations in a magnetosphere with dipole-like magnetic field is also examined.
\end{abstract}

Key words: Inhomogeneous plasma, magnetosonic waves, resonance, magnetosphere.

\section{Introduction}

The magnetosonic resonance has the same physical nature as the well-known Alfven resonance (Tamao, 1965), which in magnetospheric physics is conventionally called 'field line resonance' (Chen and Hasegawa, 1974; Radoski, 1974; Southwood, 1974). In the Alfven resonance, a monochromatic fast magnetosonic (FMS) wave propagating in an inhomogeneous plasma with magnetic field, drives an Alfven wave at the resonance magnetic shell, where its frequency is the same as the local frequency of Alfven oscillations. This coupling is due to the Alfven waves propagating practically along magnetic field lines, their frequency, for a fixed wavelength, is determined by the magnitude of the Alfven speed. Many-both theoretical (Inhester, 1987; Lee and Lysak, 1989; Leonovich and Mazur, 1989; Rankin et al., 2006), and experimental (Cheng et al., 1998; Rankin et al., 2005; Agapitov et al., 2009) (see also review by Pilipenko, 1990)—-papers have scrutinised the Alfven resonance during magnetospheric phenomena.

Slow magnetosonic (SMS) waves are in many aspects similar to Alfven waves: both the modes are guided by magnetic field lines. This results in SMS waves at the resonance magnetic shells being capable of being driven by FMS waves travelling in an inhomogeneous plasma (Yumoto, 1985). However, investigations into SMS waves in the magnetosphere are much fewer than those devoted to the Alfven resonance. Noteworthy are a significant number of papers dealing with the resonant coupling of the Alfven

Copyright (C) The Society of Geomagnetism and Earth, Planetary and Space Sciences (SGEPSS); The Seismological Society of Japan; The Volcanological Society of Japan; The Geodetic Society of Japan; The Japanese Society for Planetary Sciences; TERRAPUB.

doi:10.5047/eps.2012.07.002 and SMS waves in a curvilinear magnetic field (Southwood and Saunders, 1985; Walker, 1987; Cheng and Lin 1987; Ohtani et al., 1989; Klimushkin, 1998; Cheremnykh et al., 2004; Klimushkin and Mager, 2008).

All of these studies were performed for small-scale MHD modes with large azimuthal wave numbers $m \gg 1$. The source of such waves should be located on the same magnetic shells where they are generated. Since the magnetosphere is an opacity region for the fast magnetosonic waves with $m \gg 1$, their amplitude in the magnetosphere decreases exponentially, on a small scale, with distance from the region of its generation. The most common source of fast magnetosonic waves is believed to be either shear plasma flow at the magnetopause, or oscillations in the solar wind. Only fast magnetosonic waves with small $m \sim 1$ can penetrate inside the magnetosphere while retaining sufficient amplitude. Such waves can drive the Alfven and SMS oscillations at the resonant magnetic shells. It is exactly these resonant waves that are discussed in this paper. The Alfven resonance is a well-studied phenomenon by now, therefore we will focus on studying the magnetosonic resonance.

However, papers studying the resonance of the fast and slow magnetosonic waves are extremely few. This results, first of all, from the fact that they are difficult to detect during observations. Unlike the Alfven waves, the SMS waves are highly dissipative. The SMS wave travel speed in most of the magnetosphere is close to the thermal velocity of plasma ions to which they easily transfer their energy. There is only one exception. In the inner plasmasphere, for the magnetic shells $L<2$, where the background plasma electrons are hotter than the ions, these waves can travel almost 
without dissipation. Therefore, charged particle concentration oscillations due to the solar terminator movement over the ionosphere are regularly observed here (Afraimovich $e t$ al., 2009), with parameters allowing them to be regarded as SMS waves (Leonovich et al., 2010).

Despite-or even thanks to- the rather intensive decay of SMS waves, however, they can play an important role in their coupling with other modes of MHD oscillations, as well as interacting with background plasma ions. In solar investigations, these oscillations are often invoked for interpreting oscillations observed in the active regions (Miles and Roberts, 1989; Gonzalez and Gratton, 1991), as well as a heating mechanism for the solar corona (Nakariakov et al., 1999).

This paper will consider several problems dealing with the magnetosonic resonance and its possible signatures in the Earth's magnetosphere.

The paper is organized as follows. In Section 2, we introduce the basic equations and the equilibrium condition of the plasma configuration under consideration. Section 3 discusses the magnetosonic and Alfven resonances in a one-dimensional inhomogeneous medium in the form of a smoothly varying transition layer. This layer may be considered as a model for describing the process of FMS wave incidence and reflexion from the magnetopause and plasmapause. Section 4 solves the problem of impulse transfer from the solar wind into the geotail lobes via FMS waves. The momentum transfer to the background plasma ions is via SMS waves excited by FMS waves on the resonance magnetic shells. In Section 5, the full spatial structure of resonant SMS waves is obtained in a two-dimensionally inhomogeneous magnetosphere with a dipole magnetic field. The main features of the resonant SMS waves that can be used for their detection during observations are summarized in the Conclusion.

\section{Basic Equations}

To describe the MHD oscillation field, we will use the system of one-liquid MHD equations:

$$
\begin{aligned}
& \rho \frac{d \overline{\mathbf{v}}}{d t}=-\nabla \bar{P}+\frac{1}{4 \pi}[\operatorname{curl} \overline{\mathbf{B}} \times \overline{\mathbf{B}}], \\
& \frac{\partial \overline{\mathbf{B}}}{\partial t}=\operatorname{curl}[\overline{\mathbf{v}} \times \overline{\mathbf{B}}], \\
& \frac{\partial \bar{\rho}}{\partial t}+\nabla(\rho \overline{\mathbf{v}})=0, \\
& \frac{d}{d t} \frac{\bar{P}}{\bar{\rho}^{\gamma}}=0,
\end{aligned}
$$

where $\overline{\mathbf{B}}, \overline{\mathbf{v}}$ are the magnetic field and plasma motion velocity vectors; $\bar{\rho}, \bar{P}$ are the plasma density and pressure, $\gamma=5 / 3$ is the adiabatic constant. Assuming plasma to consist of singly-ionized hydrogen ions and electrons as well as being quasi-neutral $\left(n_{e}=n_{i} \equiv n\right)$, its parameters in the one-fluid approximation have to be understood as follows: $\overline{\mathbf{v}}=\left(m_{e} \mathbf{v}_{\mathbf{e}}+m_{i} \mathbf{v}_{\mathbf{i}}\right) /\left(m_{e}+m_{i}\right)$-mass-average velocity, $\bar{\rho}=n\left(m_{e}+m_{i}\right), \bar{P}=n\left(T_{e}+T_{i}\right)$.

We consider a disturbance due to a weak enough wave, allowing for the initial set of equations to be linearized. Let us subscript the parameters relating to unperturbed plasma with zero, leaving the wave parameters unindexed $(\bar{\rho}=$ $\left.\rho_{0}+\rho, \bar{P}=P_{0}+P, \overline{\mathbf{B}}=\mathbf{B}_{\mathbf{0}}+\mathbf{B}, \overline{\mathbf{v}}=\mathbf{v}_{\mathbf{0}}+\mathbf{v}\right)$. In the zero approximation, (1) gives the equilibrium condition for a plasma configuration in steady state $(\partial / \partial t=0)$

$$
P_{0}+\frac{B_{0}^{2}}{8 \pi}=\text { const. }
$$

\section{Magnetosonic Resonance in 1-D Inhomoge- neous Plasma}

Let us consider the problem of magnetosonic resonance in a simple one-dimensionally inhomogeneous transitional plasma layer. Such a layer-separating the magnetosphere from the solar wind-is formed at the magetopause, for example. Let us introduce a Cartesian system of co-ordinates $(x, y, z)$ for solving the problem. We will consider a plasma configuration in which the magnetic field is directed along the $z$ axis, with the plasma parameters varying in the $x$ axis direction. The $y$ axis makes it a right-hand system of coordinates. Figure 1 shows the characteristic distributions of the Alfven and SMS-wave speeds in the plasma configuration in question.

Let us denote $v_{x}=\partial \zeta / \partial t$ - the plasma velocity vector component in the $x$ direction within a wave, where $\zeta$ is the plasma element displacement. We will consider a simple harmonic wave, which in the $y$ and $z$ directions is a plane wave of the form $\exp \left(i k_{y} y+i k_{z} z-i \omega t\right)$, where $k_{y}, k_{z}$ are the respective wave vector components, $\omega$ is wave frequency. Linearizing the set of Eqs. (1)-(4) and expressing the other components of the oscillation field through $\zeta$ produces:

$$
\begin{aligned}
& v_{x}=-i \omega \zeta, \quad v_{y}=-\frac{k_{y}}{\omega} \frac{1}{K_{s}^{2}}\left(A^{2}+\frac{K_{A}^{2} S^{2}}{\chi_{S}^{2}}\right) \frac{\partial \zeta}{\partial x} \\
& v_{z}=-\frac{k_{z} K_{A}^{2} S^{2}}{\omega \chi_{S}^{2}} \frac{\partial \zeta}{\partial x} \\
& B_{x}=-i k_{z} B_{0} \zeta, \quad B_{y}=-\frac{k_{z} B_{0}}{\omega} v_{y}, \\
& B_{z}=-\frac{K_{A}^{2} B_{0}}{\chi_{s}^{2}}\left(1-\frac{k_{z}^{2} S^{2}}{\omega^{2}}\right) \frac{\partial \zeta}{\partial x}-\frac{d B_{0}}{d x} \zeta \\
& P=-\gamma P_{0} \frac{K_{A}^{2}}{\chi_{S}^{2}} \frac{\partial \zeta}{\partial x}+\frac{1}{8 \pi} \frac{d B_{0}^{2}}{d x} \zeta
\end{aligned}
$$

where

$$
\begin{aligned}
& K_{A}^{2}=1-\frac{k_{z}^{2} A^{2}}{\omega^{2}}, \quad K_{s}^{2}=K_{A}^{2}-\frac{k_{y}^{2} A^{2}}{\omega^{2}}, \\
& \chi_{S}^{2}=1-\frac{k_{y}^{2}+k_{z}^{2}}{\omega^{2}}\left(A^{2}+S^{2}-\frac{k_{z}^{2} A^{2} S^{2}}{\omega^{2}}\right),
\end{aligned}
$$

$A=B_{0} / \sqrt{4 \pi \rho_{0}}$ is the Alfven speed, $S=\sqrt{\gamma P_{0} / \rho_{0}}$ is sound speed in plasma. For displacement $\zeta$, we have the equation

$$
\frac{\partial}{\partial x} \frac{\rho_{0} \Omega^{2}}{k_{x}^{2}} \frac{\partial \zeta}{\partial x}+\rho_{0} \Omega^{2} \zeta=0,
$$

where $\Omega^{2}=\omega^{2}-k_{z}^{2} A^{2}$,

$$
k_{x}^{2}=-k_{y}^{2}-k_{z}^{2}+\frac{\omega^{4}}{\omega^{2}\left(A^{2}+S^{2}\right)-k_{z}^{2} A^{2} S^{2}} .
$$




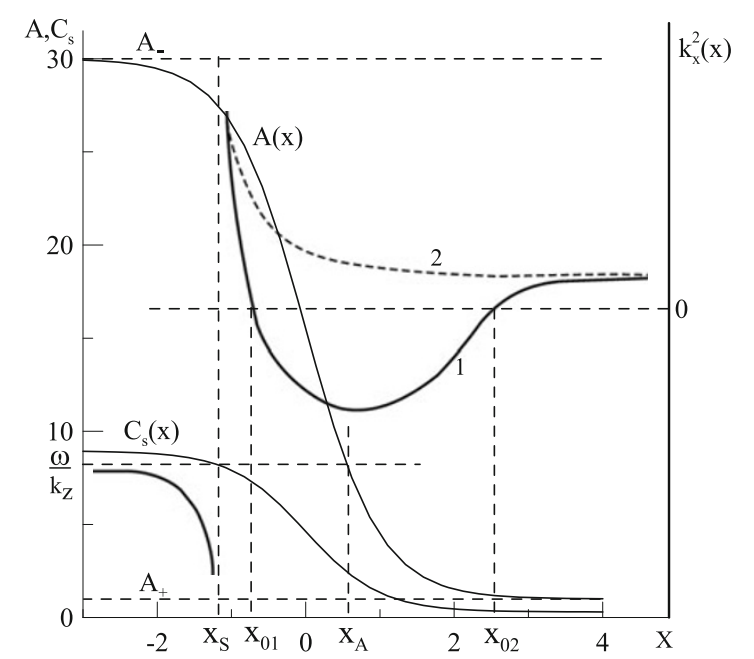

Fig. 1. Profiles of Alfven speed $A(x)$, SMS wave speed $C_{s}(x)$ and square of the wave vector WKB component $k_{x}^{2}(x)$ across the transition layer.

It is evident from (9) that, in the WKB approximation, $k_{x}^{2}$ is the $x$ component squared of the wave vector when the solution can be presented as $\zeta \sim \exp \left(i \int k_{x} d x\right)$.

The behaviour of the $k_{x}^{2}(x)$ function is important for the problem to be stated correctly. We wish to explore the process of incidence and reflexion of a magnetosonic wave on a smoothly varying transition layer. If the wave source is to the right of the transition layer, the solution of the problem should be the superposition of the incident and reflected waves of finite amplitude when $x \rightarrow \infty$. Two variants of the distribution of the $k_{x}^{2}(x)$ function are possiblenumbered 1 and 2 in Fig. 1. An analysis of (10) reveals that, for monotone increasing $A(x)$, when the $x$ coordinate varies from $+\infty$ to $-\infty$, the $k_{x}^{2}(x)$ function passes through zero twice at points which we will denote as $x_{01}, x_{02}$, between which the opacity region (where $k_{x}^{2}(x)<0$ ) is located. This behaviour of $k_{x}^{2}(x)$ is illustrated by curve 1 in Fig. 1.

There are also two singular points in Eq. (9) at which the coefficient attached to the higher derivative tends to zero. One is the Alfven resonance point, $x_{A}$, defined by equality $\Omega^{2}\left(x_{A}\right)=0$, located in the opacity region in the interval $\left(x_{01}, x_{02}\right)$. The second is the point of magnetosonic resonance, $x_{S}$, where the denominator in expression (10) tends to zero, producing the local dispersion equation for SMS waves when $\left|k_{x}^{2}\right| \rightarrow \infty: \omega^{2}=k_{z}^{2} C_{s}^{2}\left(x_{S}\right)$, where $C_{s}^{2}=A^{2} S^{2} /\left(A^{2}+S^{2}\right)$. The point $x_{S}$ is located more to the left of the turning point $x_{01}$, the transparency region for SMS waves being located between the two. To the left of $x_{S}$, there is an opacity region expanding to $-\infty$ in the $x$ direction.

Curve 2 in Fig. 1 corresponds to the case when the transparency region for SMS waves extends up to $\infty$, and there is no resonance surface for the Alfven waves. Analyzing expression (10) helps define the two ranges of wave field and plasma parameters corresponding to curves 1 and 2 in Fig. 1, when $k_{x}^{2}(\infty)>0$ :

$$
\frac{\omega^{2}}{k_{z}^{2} A_{+}^{2}}>\omega_{A 1}^{2}, \quad \frac{\beta^{*}}{1+\beta^{*}}<\frac{\omega^{2}}{k_{z}^{2} A_{+}^{2}}<\omega_{A 2}^{2},
$$

where $\beta^{*}=S^{2} / A^{2}$, and $\omega_{A 1}^{2}, \omega_{A 2}^{2}$ are the two roots of the biquadratic equation

$$
\omega_{A}^{4}-\left(1+k_{y}^{2} / k_{z}^{2}\right)\left[\omega_{A}^{2}\left(1+\beta^{*}\right)-\beta^{*}\right]=0,
$$

corresponding to the solutions with the positive/negative radical. The first describes a FMS wave, and the second a SMS wave incident on the transition layer.

Let us describe the Alfven speed profile $A(x)$ as a function of the form

$$
A(x)=\frac{1}{2}\left[A_{-}+A_{+}+\left(A_{-}-A_{+}\right) \tanh \left(\frac{x}{\Delta}\right)\right],
$$

where $A_{ \pm}$is the Alfven speed as $x \rightarrow \pm \infty, \Delta$ is the typical thickness of the transition layer. In the chosen model of the Alfven speed $A_{+}=1$ and $\Delta=1$ (see Fig. 1). The ratio $A_{-} / A_{+}=30$ is chosen large enough for both types of resonant surfaces - those for the Alfven and SMS waves - to be able to exist in this system, for a wide enough spread of the plasma temperature values (from $\beta^{*}=0.01$ to $\beta^{*} \sim 1$ ).

Let us define the solution of (9) near the resonant surface $x=x_{S}$. Let us linearize the coefficient attached to the higher derivative in (9), representing $k_{x}^{-2} \approx a_{s}^{2} \xi_{s}$, where $\xi_{s}=\left(x-x_{S}\right) / a_{s}, a_{s}=\left(\partial k_{x}^{-2} / \partial x\right)_{x=x_{s}}$ is the characteristic scale of the $k_{x}^{-2}$ variation near $x=x_{S}$. In order to regularize the singularity in the solution of (9) we will formally introduce a damping decrement, $\gamma_{s}$, for SMS waves near the resonant surface $x=x_{S}$, making a substitution, $\omega \rightarrow \omega+i \gamma_{s}$, in the denominator of (10). Equation (9) may then be rewritten as

$$
\frac{\partial}{\partial \xi_{s}}\left(\xi_{s}+i \varepsilon_{s}\right) \frac{\partial \zeta}{\partial \xi_{s}}+\zeta=0
$$

near $x=x_{S}$, where $\varepsilon_{s}=k_{y} a_{s} \gamma_{s} / k_{z} C_{s}\left(x_{s}\right)$ is the regularized factor determined by the decrement of SMS waves, where the subscript ${ }_{S}$ indicates that the values of the parameters are taken for $x=x_{S}$. Its solution is

$$
\zeta=C_{1} I_{0}\left(2 \sqrt{-\xi_{s}-i \varepsilon_{s}}\right)+C_{2} K_{0}\left(2 \sqrt{-\xi_{s}-i \varepsilon_{s}}\right)
$$

where $I_{0}(z), K_{0}(z)$ is the modified Bessel functions. If the wave source is to the right of the transition layer, then, using an asymptotic representation of (14) in the opacity region at $\xi_{s} \rightarrow-\infty\left(K_{0}(z) \rightarrow \sqrt{\pi / 2 z} e^{-z}, I_{0}(z) \rightarrow e^{-z} / \sqrt{2 \pi z}\right)$, we find $C_{1}=0$. To the right of resonance point $\xi_{s}=0$ the solution (14) has the form

$$
\zeta=-i \frac{C_{2} \pi}{2} H_{0}^{(2)}\left(2 \sqrt{\xi_{s}+i \varepsilon_{s}}\right)
$$

where $H_{0}^{(2)}(z)$ is the Hankel function of the second kind, which, as $\xi_{s} \rightarrow \infty$, has an asymptotic representation, $H_{0}^{(2)}\left(2 \sqrt{\xi_{s}}\right) \approx \pi^{-1 / 2} \xi_{s}^{-1 / 4} \exp \left(-i 2 \sqrt{\xi_{s}}+i \pi / 4\right)$. When $\xi_{s} \rightarrow 0$, the solution (14)-(15) is

$$
\zeta=-\frac{C_{2}}{2} \ln \left(-\xi_{s}-i \varepsilon_{s}\right)
$$

i.e. it has the same logarithmic singularity on the resonance surface, as in the region of the Alfven resonance. 
There are essential differences, however. The above solution describes a wave incident on the resonance surface. The wave reflected from this surface is absent-it is described by function $I_{0}(z)$, whose coefficient $C_{1}=0$. This means that the total energy of a wave incident on the resonance surface is completely absorbed in its neighbourhood, irrespective of the dissipation mechanism involved. If the transparency region for SMS waves expands ad infinitum to the right (which corresponds to the second condition (11)), such waves will be completely absorbed near the resonance surface. If the parameters of the wave under consideration are such (the first condition (11)) that the incident wave "leaks" through the barrier of opacity region $\left(x_{01}, x_{02}\right)$ into the SMS-wave transparency region $\left(x_{s}, x_{01}\right)$, then the energy of the wave penetrating through this barrier is absorbed completely in the neighbourhood of the resonance surface. Note the energy absorption of the incident wave does not exceed $50 \%$ in the neighbourhood of the Alfven resonance surface located deep within the opacity region $\left(x_{01}, x_{02}\right)$ (Leonovich et al., 2010).

If the wave source is to the left of the resonance surface (in the opacity region $x<x_{s}\left(\xi_{s}<0\right)$ ), then in the SMS wave transparency region, $\xi_{s}>0$, expanding ad infinitum, the solution of (9) must describe a wave escaping from the resonance surface. There is no incident wave on the resonance surface, as described by the function $K_{0}(z)$, to the right of $\xi_{s}=0: C_{2}=0$. Since the function $I_{0}(z)$ is regular on the resonance surface, magnetosonic resonance is also absent.

Using the ideal MHD approximation to study the SMS waves, their very high dissipation ought to be taken into account. To this end, their decrement can be introduced in equations, near the resonance surfaces for SMS waves as was done above. The magnitude of the decrement crucially depends on the plasma ion to electron temperature ratio. The dependence of the SMS wave decrement on the plasma nonisothermality level is specified in Appendix A based on the kinetic theory equations.

Detecting the presence of resonant SMS waves in a plasma of high dissipation level is a rather difficult problem. The oscillation amplitude increases only little at the resonance surface. There is another possibility, however. Let the parameters of the FMS wave incident on the inhomogeneous plasma layer be such that the resonance surfaces for both the Alfven and SMS waves exist simultaneously. Due to weakly localized resonant SMS oscillations, they canwhen dissipation is large enough-affect the behaviour of oscillations near the Alfven resonance surface. The presence of magnetosonic resonance in the plasma system under study may be detected via the behaviour of the oscillation hodograph when moving through these surfaces.

A distinctive feature of resonant Alfven oscillations is the change of the hodograph rotation direction of the transverse magnetic field vector $\mathbf{B}_{\perp}=\left(B_{x}, B_{y}\right)$ as we pass through the resonant surface. It follows from the sign reversal in $\partial \zeta / \partial x$. In a case with small decrements $\gamma_{A}, \gamma_{s} \ll \omega$, this rule is valid when we pass through each resonant surface $x=x_{A}$ and $x=x_{S}$. We will look, however, at what happens when decrements $\gamma_{A}$ and $\gamma_{S}$ are not too small.

Figure 2 demonstrates the distribution of $\partial \zeta / \partial x$ as cal-

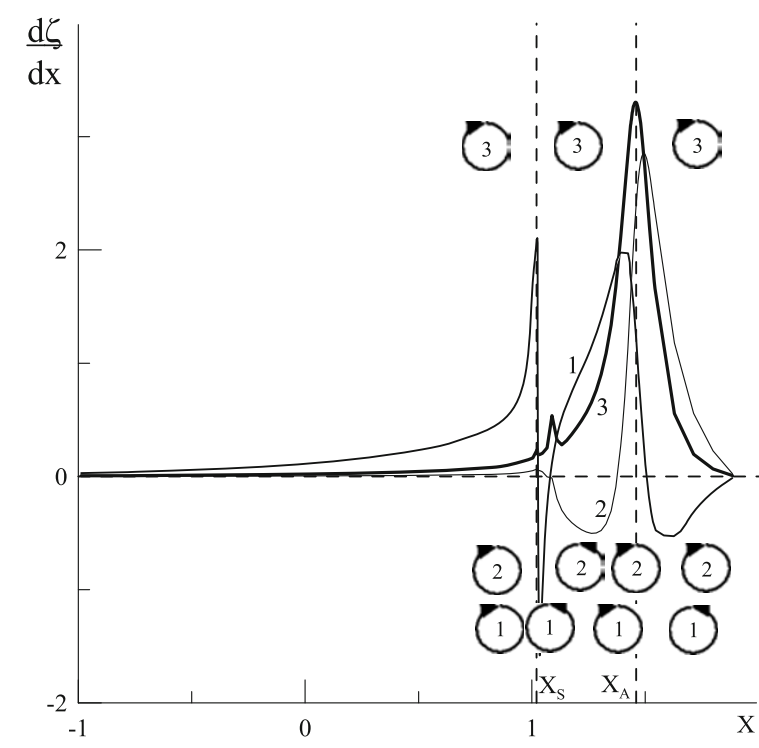

Fig. 2. Rotation of the polarisation hodograph of resonant MHD oscillations in the neighbourhood of resonant surfaces $x_{A}$ and $x_{S}$ for different dissipation levels in the Alfven $\left(\varepsilon_{A}=0.1\right)$ and SMS oscillations. Notations 1, 2 and 3 correspond to different dissipation rates of the SMS oscillations: $1-\left|\bar{\varepsilon}_{s}\right|=0.01,2-\left|\bar{\varepsilon}_{s}\right|=0.1,3-\left|\bar{\varepsilon}_{s}\right|=1$.

culated for $\varepsilon_{A}=\gamma_{A} / \omega=0.1$ and three values of $\varepsilon_{s}=$ $\gamma_{s} / \omega=0.01 ; 0.1 ; 1$. Here the behaviour of the hodographs is conventionally presented in the plane $\left(B_{y}, B_{x}\right)$. For small $\varepsilon_{s}=0.01\left(T_{e} / T_{i} \gg 1\right)$ the behaviour of the hodograph is as expected. When $\varepsilon_{s}$ increases to $0.1\left(T_{e} \sim T_{i}\right)$ the points where the hodograph rotation direction changes shift away from the resonant surfaces about as far as the distance between them. With $\varepsilon_{s}$ increasing further to $1\left(T_{e} / T_{i} \approx 0.1\right)$, the hodograph rotation direction does not change at all. This example demonstrates that the presence in the system of strongly damped resonant SMS oscillations can change the behaviour of the field components essentially, even in the neighbourhood of the resonant surface for the Alfven waves.

Almost everywhere in the Earth's magnetosphere, $T_{e} \ll$ $T_{i}$. As follows from the above calculations, this means that SMS waves decay fast $\left(\gamma_{s} \sim \omega\right)$. Therefore, the resonance peaks are poorly-expressed for these waves and difficult to detect in observations. If the resonance peaks for the Alfven waves are also present in the system, however, the above features of the hodograph behavior may indicate the presence of resonant SMS waves. The only exception is the region inside the plasmasphere on the magnetic shells $L<2$, where $T_{e} \approx 2 T_{i}$ (Titheridge, 1998). The decrement of SMS waves is relatively small here so that resonant SMS oscillations can be observed directly. It is, however, hard to imagine FMS waves capable of reaching magnetic shells that are so close to Earth.

\section{Transfer of Momentum from the Solar Wind Into Geotail via Magnetosonic Waves}

Let us now consider the specific problem of momentum transfer from the solar wind into geotail lobes via magnetosonic waves.

The magnetosheath plasma flow is turbulent. Such 


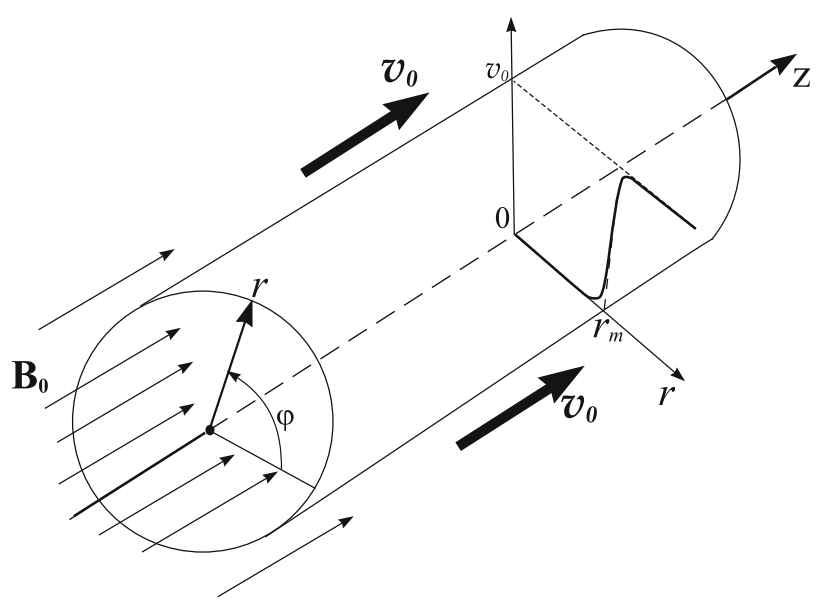

Fig. 3. A cylindrical model of the magnetotail within the solar wind plasma flow. Magnetic field $\mathbf{B}_{\mathbf{0}}$ is along the plasma cylinder axis. The radial distribution of the solar wind velocity $v_{0}$ flowing round the geotail is shown schematically.

plasma oscillations can be regarded as a stochastic flow of magnetosonic waves, partly directed towards the magnetosphere. Leonovich et al. (2003) have shown that up to 50\% of the energy of this wave flux can penetrate into the geotail. The integrated energy of the wave flux penetrating into the magnetosphere during a typical time interval between two successive substorms is two orders of magnitude larger than the total energy of magnetospheric convection and can be used to maintain it. This is only a potential capacity, however.

For each harmonic of magnetosonic waves there is a surface in the magnetosphere from which it is completely reflected. Therefore, if no appreciable absorption of their energy takes place while magnetosonic oscillations travel from the magnetopause to the turning point, they must be reflected back into the solar wind in almost their entirety. The energy of MHD oscillations is known to be efficiently absorbed at resonance surfaces for the Alfven and slow magnetosonic waves (Leonovich and Kozlov, 2009). The SMS waves are especially interesting in this regard. Due to their very dissipative nature they are weakly localized across magnetic shells and can interact with ions of the bulk of the background plasma distribution function. To check this possibility, we employ quasilinear theory to calculate the velocity the background plasma acquires when interacting with the flux of fast magnetosonic waves penetrating into the magnetosphere from the magnetosheath.

Let us consider a model magnetotail in the form of an inhomogeneous plasma cylinder as shown in Figs. 3-4. The plasma distribution over radius corresponds to the geotail lobes. This model does not explicitly take into account the plasma sheet. Its presence is simulated by the radial distribution of the Alfven and SMS velocity. Moving away from the cylinder axis, they change from values characteristic of the plasma sheet to those typical of the geotail lobes. The radial distribution of the Alfven speed is plotted in Mazur and Leonovich (2006) based on satellite data for the distribution of plasma concentration and magnetic field in the magnetosphere (Sergeev and Tsyganenko, 1980; Borovsky

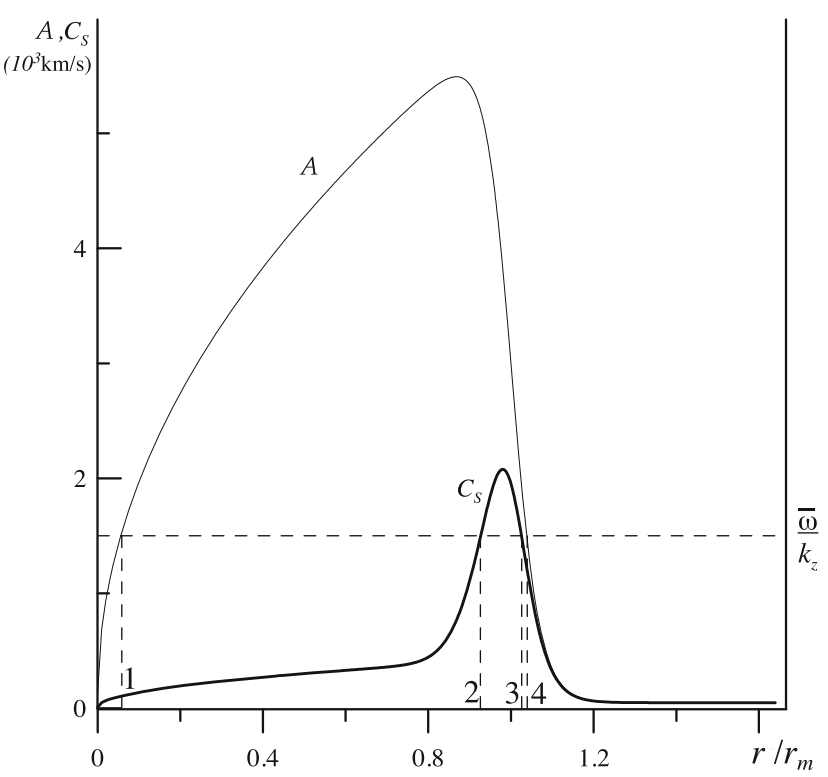

Fig. 4. Distribution of the Alfven speed $A(r)$ and SMS wave speed $C_{s}(r)$ in the magnetotail and in the solar wind. On the resonance shells $r=r_{S}$ (points 2 and 3) and $r=r_{A}$ (points 1 and 4), the parallel phase velocity $\bar{\omega} / k_{z}$ of a monochromatic wave is equal to, respectively, local speed $C_{S}$ of SMS waves and Alfven speed $A$.

et al., 1998). Since the main results of that study concern the region of open field lines, the presence of plasma sheet should not be an essential element in the calculations.

We introduce a cylindrical coordinate system $(r, \phi, z)$ in which the origin $r=0$ coincides with the axis of the plasma cylinder. The background magnetic field is directed along the $z$ axis. We assume that plasma in the magnetosheath moves along the $z$ axis at velocity $v_{0}$, while plasma is motionless in the geotail, in the absence of waves (see Fig. 3 ). Transition from the magnetospheric parameters to the magnetosheath parameters occurs in a narrow transition layer of thickness $\Delta_{r} \ll r_{m}$, where $r_{m}$ is the characteristic radius of the geotail. We set such a plasma density distribution over the radius that its maximum is reached on the axis of the plasma cylinder, falling to a minimum toward its boundary. Magnetic field in the magnetotail is stronger than in the solar wind. The distribution of the Alfven speed $A=B_{0} / \sqrt{4 \pi \rho_{0}}$ over the radius is presented in Fig. 4. Such a distribution is typical of plasma parameters in the geotail lobes.

As can be seen here, a maximum concentration of resonance surfaces for SMS waves in the geotail lobes is reached near the transitional layer. Using the same system of MHD equations (1)-(4) for cylindrical harmonics of the form $\exp \left(i k_{z} z+i m \phi-i \omega t\right)$, where $m=0,1,2,3 \ldots$ is the azimuthal wave number, produces the following equation for displacement $\zeta$ :

$$
\frac{\partial}{\partial r} \frac{\rho_{0} \Omega^{2}}{k_{r}^{2}} \frac{1}{r} \frac{\partial r \zeta}{\partial r}+\rho_{0} \Omega^{2} \zeta=0
$$

where $\Omega^{2}=\bar{\omega}^{2}-k_{z}^{2} A^{2}, \bar{\omega}=\omega-k_{z} v_{0}$ is the Doppler- 

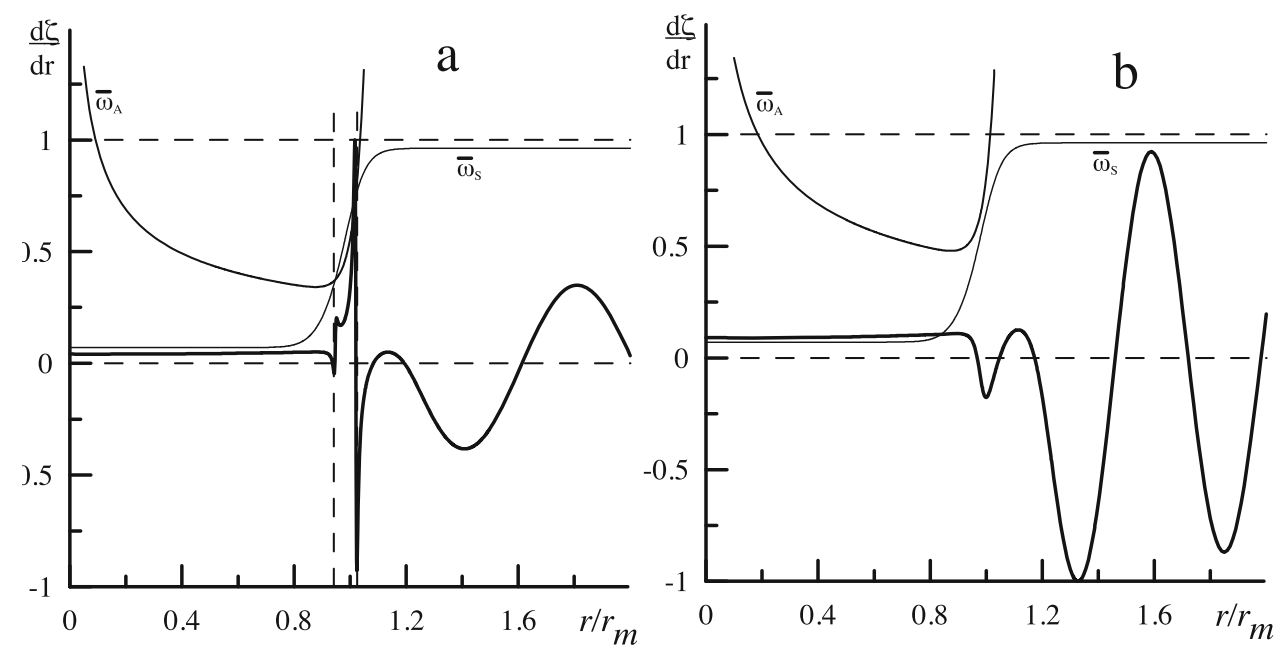

Fig. 5. The spatial structure of monochromatic MHD waves for azimuthal harmonic $m=1$ with different values of parallel phase velocity $\bar{\omega} / k_{z}$ : (a) oscillations for which there are resonance shells for SMS waves in the geotail $\left(\bar{\omega}_{A}\left(r_{S}\right)=\bar{\omega}_{S}\left(r_{S}\right)\right)$, (b) oscillations with no resonance shells inside the geotail.

modified oscillation frequency,

$$
\begin{aligned}
k_{r}^{2} & =\frac{\bar{\omega}^{4}}{\bar{\omega}^{2}\left(A^{2}+S^{2}\right)-k_{z}^{2} A^{2} S^{2}}-k_{z}^{2}-\frac{m^{2}}{r^{2}} \\
& =\frac{k_{z}^{2}}{1+\beta^{*}} \frac{\left(\bar{\omega}_{A}^{2}-\bar{\omega}_{A 1}^{2}\right)\left(\bar{\omega}_{A}^{2}-\bar{\omega}_{A 2}^{2}\right)}{\left(\bar{\omega}_{A}^{2}-\bar{\omega}_{S}^{2}\right)}
\end{aligned}
$$

and $\bar{\omega}_{A}=\bar{\omega} / k_{z} A(r), \bar{\omega}_{S}=\sqrt{\beta^{*} /\left(1+\beta^{*}\right)}, \beta^{*}=S^{2} / A^{2}$. We substitute $\bar{\omega} \rightarrow \bar{\omega}+i \gamma_{s}$ in the denominator of (18) to take into account SMS wave dissipation.

Figure 5 shows the radial structure of two monochromatic harmonics. There are resonance surfaces for SMS waves in the magnetosphere (Fig. 5(a)) for one of them, but not for the other (Fig. 5(b)). This figure presents the unitynormalized structure of the derivative $d \zeta / d r$ determining the maximum oscillation amplitude on the resonance surfaces. The resonance surfaces for the SMS wave are determined by the intersection points of functions $\operatorname{Re}\left(\bar{\omega}_{\mathrm{A}}(\mathrm{r})\right)$ and $\bar{\omega}_{S}(r)$, where the real part of the denominator in (10) vanishes. As was noted above, the decrement of SMS waves strongly depends on the plasma ion to electron temperature ratio. In the solar wind the plasma electrons are hotter than the ions $\left(T_{e} \approx 3 T_{i}\right)$, therefore, we assume $\bar{\gamma}_{S} \approx 10^{-2} \omega$ in the magnetosheath. In the tail lobes, on the contrary, the plasma ions are hotter than the electrons $\left(T_{i} \approx 8 T_{e}\right)$, which corresponds to $\bar{\gamma}_{S} \approx 0.8 \omega$ (see Appendix A). The decrement of SMS waves chosen for the magnetosheath changes to the one typical of the magnetosphere in the same transition layer as the other plasma parameters.

Consider the problem of the ion distribution function in magnetospheric plasma transformed under the impact of the MHD wave flux from the magnetosheath. We use kinetic theory equations in the locally quasilinear approximation. This means that, on each resonance shell inside the geotail, we will consider the distribution function in the same manner as if it were defined over the entire space, while assuming the MHD oscillation field to correspond to this shell. We assume the plasma to consist of the hydrogen ions and electrons. The asymptotic (when $t \rightarrow \infty$ ) equation for the ion distribution function $f\left(v_{\|}\right)$in the presence of SMS waves has the following form (see Appendix B)

$$
\frac{\partial \bar{f}}{\partial t} \approx \frac{\partial}{\partial v_{\|}} \bar{D} \frac{\partial \bar{f}}{\partial v_{\|}}
$$

where we use the Maxwell distribution function

$$
\bar{f}\left(v_{\|}, t=0\right)=\frac{n_{0}}{\sqrt{\pi} v_{T i}} \exp \left(-\frac{v_{\|}^{2}}{v_{T i}^{2}}\right),
$$

as the initial condition for solving (19). Presumably, the plasma acquires this state in the geomagnetic tail lobes during the substorm recovery phase. Here $n_{0}$ is the plasma ion concentration, $v_{T i}=\sqrt{2 T_{i} / m_{i}}$ is the thermal velocity of plasma ions on the magnetic shell under scrutiny,

$$
\begin{aligned}
\bar{D}= & \frac{\pi}{2} \frac{v_{T i}^{4}}{v_{\|} B_{0}^{2}} \sum_{m=0}^{\infty} \\
& \times \int_{0}^{\infty}\left\langle\left|\nabla_{r} \bar{B}_{r}\left(r, m, k_{z}=\omega / v_{\|}, \omega\right)\right|^{2}\right\rangle d \omega
\end{aligned}
$$

is the ion diffusion coefficient in the velocity space, $v_{\|}$is ion velocity along magnetic field lines, $\left\langle\left|B_{r}\right|\right\rangle$ is the averaged amplitude of the radial magnetic field of oscillation, $m$ is azimuthal wave number.

Multiplying (19) by $\bar{f}$ on the left and integrating over $v_{\|}$ yields

$$
\frac{1}{2} \frac{\partial}{\partial t} \int_{-\infty}^{\infty} \bar{f}^{2} d v_{\|} \approx-\int_{-\infty}^{\infty} \bar{D}\left(\frac{\partial \bar{f}}{\partial v_{\|}}\right)^{2} d v_{\|} .
$$

Hence, if a new equilibrium state is reached $(\partial \bar{f} / \partial t=0)$ at the asymptotic $t \rightarrow \infty$, a "plateau" must appear in the distribution function $\left(\partial \bar{f} / \partial v_{\|}=0\right)$ in the intervals of $v_{\|}$ where $\bar{D} \neq 0$.

There are three areas where a "plateau" forms in the distribution function $\bar{f}:-C_{S \max }<v_{\|}<-C_{S \min }, C_{S \min }<$ $v_{\|}<v_{2}$ and $v_{1}>v_{\|}>C_{S \max }$. The $C_{S \max }, C_{S \text { min }}$ correspond to the maximum and minimum velocities of the SMS 


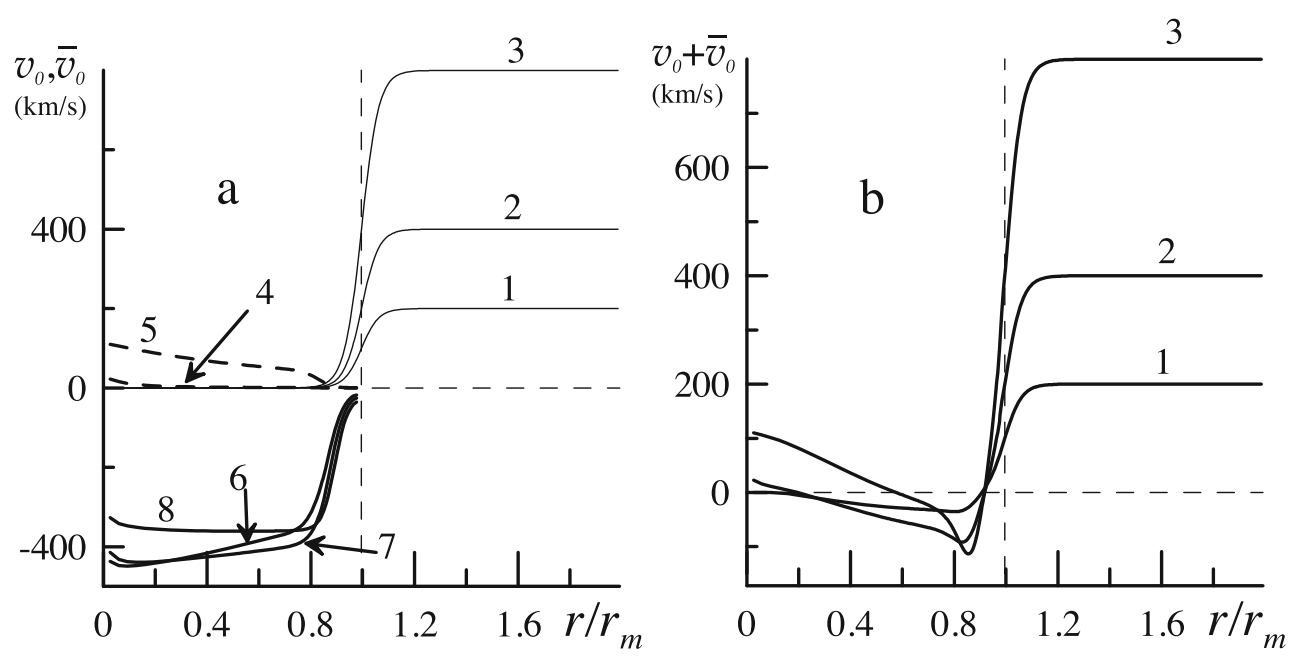

Fig. 6. Distribution over the radius of the plasma flow velocities $v_{0}$ in the magnetosheath and magnetospheric convection velocity $\bar{v}_{0}$ for different velocities of the solar wind. Curves 1, 2, 3 in panel (a) correspond to the solar wind velocity distribution for $v_{0}=200,400,800 \mathrm{~km} / \mathrm{s}$, curves 4 and 5 are the magnetospheric convection velocities when $v_{0}=400,800 \mathrm{~km} / \mathrm{s}$, in the absence of "upstream" FMS waves in the magnetosheath, and curves $6,7,8\left(v_{0}=200,400,800 \mathrm{~km} / \mathrm{s}\right)$ are the magnetospheric convection velocities for equal fluxes of "downstream" and "upstream" FMS waves in the magnetosheath. Panel (b) shows the distribution of complete velocity $v_{0}+\bar{v}_{0}$ (curves $1,2,3$ for $v_{0}=200,400,800 \mathrm{~km} / \mathrm{s}$ ) when the flux of "downstream" FMS waves prevails over that of the "upstream" FMS waves in the magnetosheath.

waves for which the local dispersion equation $\omega^{2}=k_{z}^{2} C_{S}^{2}$ holds true in our model. The value $C_{S \min } \approx 8 \mathrm{~km} / \mathrm{s}$ is reached on the axis of the plasma cylinder, $C_{S \max } \approx 2000$ $\mathrm{km} / \mathrm{s}$ in the vicinity of the magnetopause. The solar wind is transparent in the intervals of parallel wave numbers $k_{z}<$ $\min \left(k_{1}, k_{2}\right)$ and $k_{z}>\max \left(k_{1}, k_{2}\right)$, where $k_{1,2}=\omega / v_{1,2}$, $v_{1}=v_{0}+S_{w}, v_{2}=v_{0}-S_{w}, S_{w}$ is sound velocity in the solar wind. In our model of the medium the sound velocity in the magnetosheath $S_{w}=177 \mathrm{~km} / \mathrm{s}$. For solar wind plasma flows with $v_{0}>200 \mathrm{~km} / \mathrm{s}$ we have $v_{1}>v_{2}>0$ and the solar wind is opaque when $0<k_{1}<k_{z}<k_{2}$. Considering the resonance conditions for plasma particles interacting with waves $\left(k_{z}=\omega / v_{\|}\right)$, we find that the distribution function remains unchanged in the range $v_{2}<v_{\|}<v_{1}$. The area $C_{S \text { min }}<v_{\|}<v_{2}$ corresponds to "downstream", while the two other areas to "upstream" FMS waves in the solar wind.

The level of the plateau in each of these areas is determined by the condition that the total number of particles should remain the same and can be expressed through the following relation

$$
\bar{f}_{j}=\int_{v_{\| \min }}^{v_{\| \max }} \bar{f}\left(v_{\|}\right) d v_{\|} /\left(v_{\| \max }-v_{\| \min }\right),
$$

where $j=1,2,3$ is the number of an area with a "plateau", and the values $v_{\| \max \text {,min }}$ correspond to the maximum and minimum value of the parallel velocity of particles in each of these intervals.

The average velocity of plasma resulting from its interaction with MHD waves is determined by the equation

$$
\bar{v}_{0}=\frac{1}{n_{0}} \int_{-\infty}^{\infty} v_{\|} \bar{f}\left(v_{\|}\right) d v_{\|} .
$$

Obviously, the contribution from symmetric (with respect to $v_{\|}=0$ ) parts of $\bar{f}\left(v_{\|}\right)$is zero. Figure 6 shows the distribution of $v_{0}(r)$ as calculated for the parameters of the cylindrical model of the geotail we use in this study, for different solar wind velocities in the magnetosheath.

Figure 6(a) presents the solar wind velocity $v_{0}(r)$ profiles taking into account the transition layer, and plasma velocity profiles in the geotail lobes $\bar{v}_{0}(r)$ calculated for two limiting cases. The first of these (curves 4 and 5 in Fig. 6(a)) assumes that all waves in the magnetosheath move "downstream" and no plateaux form in the ranges $-C_{S \max }<v_{\|}<-C_{S \text { min }}$ and $v_{1}>v_{\|}>C_{S \text { max }}$. Obviously, in this case the impulse transferred by MHD waves to ions in the geotail lobes is tailward $\bar{v}_{0}(r)>0$. In the second limiting case, the "downstream" and "upstream" fluxes of waves are equal. It is evident from Fig. 5(a), that in this case the impulse transferred to plasma ions is Earthward. From satellite observations of solar wind oscillations, it is difficult to determine which portion of the wave flux is "downstream" or "upstream".

The most probable seems to be an intermediate case between the two, when the "downstream" waves in the magnetosheath occupy a broader part of the spectrum than do the "upstream" waves. The summarized plasma velocity distribution $v_{0}(r)+\bar{v}_{0}(r)$ in the case when the "upstream" waves are absent from the range $-C_{S \max }<-300 \mathrm{~km} / \mathrm{s}<$ $v_{\|}<-C_{S \min }$ is displayed in Fig. 6(b). Evidently, in this case the impulse transferred to ions in the regions adjacent to the transition layer reverses the plasma flow motion back to Earth, whereas closer to the cylinder axis the motion becomes tailward again. Note that the model in question is inapplicable to those inner parts of the geotail where the plasma sheet lies. As will be seen later, another reason why the obtained results cannot be used for the inner regions of the geotail is that the characteristic time for the asymptotic regime of the plasma flow to set in there is too long.

The characteristic time $\tau$ needed for the completely motionless plasma to switch to the asymptotic regime of its motion is determined by the amplitude of MHD waves transferring the momentum from the solar wind into the 
magnetosphere. To estimate this time, let us replace the time derivative in (22) with $\tau^{-1}$, resulting in

$$
\tau \approx 2 \int_{-\infty}^{\infty} \bar{f}^{2} d v_{\|} / \int_{-\infty}^{\infty} \bar{D}\left(\frac{\partial \bar{f}}{\partial v_{\|}}\right)^{2} d v_{\|}
$$

For the $\bar{f}$ function, let us choose the Maxwell distribution of the form (20).

To calculate the diffusion coefficient $\bar{D}$, it is necessary to specify the spectrum of MHD waves in the magnetosheath. There are many onboard observations of variations in the solar wind parameters. They are generally stochastic oscillations with a "white noise" spectrum (Matthaeus and Goldstein, 1982). Here we shall use the model spectrum function constructed in Leonovich and Mishin (1999)

$$
\left\langle\left|\bar{B}_{r}\right|^{2}\right\rangle=C \Phi\left(k_{t}, \omega\right) \omega^{-\alpha} k_{t}^{-2 \beta},
$$

where $C$ is a constant determined by the average oscillation amplitude, and $\Phi\left(k_{t}, \omega\right)$ is a step function determining the upper and lower limits of the spectrum, as well as the wave range for which the solar wind is an opacity region. This function well describes properties of the spectrum of magnetosonic waves observed in the solar wind (Matthaeus and Goldstein, 1982; Marsh and Tu, 1990; Goldstein et al., 1995). The function $\Phi\left(k_{t}, \omega\right)$ may be written as

$$
\begin{aligned}
\Phi\left(k_{t}, \omega\right)= & \Theta(\omega-\hat{\omega}) \Theta\left(\omega_{i}-\omega\right) \\
& \times\left[\Theta\left(\bar{k}_{z}-\bar{k}_{2}\right)+\Theta\left(\bar{k}_{1}-\bar{k}_{z}\right)\right],
\end{aligned}
$$

where $\Theta(x)$ is the Heaviside step function, and $\bar{k}_{1}=$ $-\omega / S_{w}<\bar{k}_{z}<\bar{k}_{2}=\omega / S_{w}$ is the range of parallel wave numbers corresponding to solar wind opacity for FMS waves. Constant $C$ in (24) is determined by the inverse Fourier transformation

$$
\begin{aligned}
\left\langle\left|B_{r}\right|^{2}\right\rangle= & \frac{1}{(2 \pi)^{3 / 2}} \sum_{m=0}^{\infty} \int_{0}^{\infty} d \omega \int_{-\infty}^{\infty}\left\langle\left|\bar{B}_{r}\right|^{2}\right\rangle d \bar{k}_{z} \\
= & \frac{C}{(2 \pi)^{3 / 2}} \sum_{m=0}^{\infty} \int_{0}^{\infty} \omega^{-\alpha} d \omega \\
& \times \int_{-\infty}^{\infty} \Phi\left(k_{t}, \omega\right) k_{t}^{-2 \beta} d \bar{k}_{z},
\end{aligned}
$$

where $\left\langle\left|B_{r}\right|^{2}\right\rangle$ is the mean square of the amplitude of the $B_{r}$ component of the solar wind oscillation field at $r=2 r_{m}$. In our calculations we assume $\left\langle\left|B_{r}\right|\right\rangle \sim 0.2 B_{0} \approx 1 \mathrm{nT}$.

Figure 7 shows the distribution over the radius of the characteristic time $\tau$ needed for the asymptotic regime to set in in the geotail plasma flow, as calculated using formula (23), in which the diffusion coefficient is determined by (21), and the spectrum of FMS fluctuations in the magnetosheath (24) corresponds to the plasma flow profiles in Fig. 6(b). It is evident that the values of $\tau$ comparable with the time during which the geotail can be regarded as a stable enough plasma configuration (average interval between two successive substorms $\sim 3-6 \mathrm{~h}$ ) is achieved in the ranges $0.8 r_{m}<r<r_{m}$ (for the $v_{0}=400 \mathrm{~km} / \mathrm{s}$ solar wind) and $0.85 r_{m}<r<r_{m}$ (for the $v_{0}=200,800 \mathrm{~km} / \mathrm{s}$ solar wind).

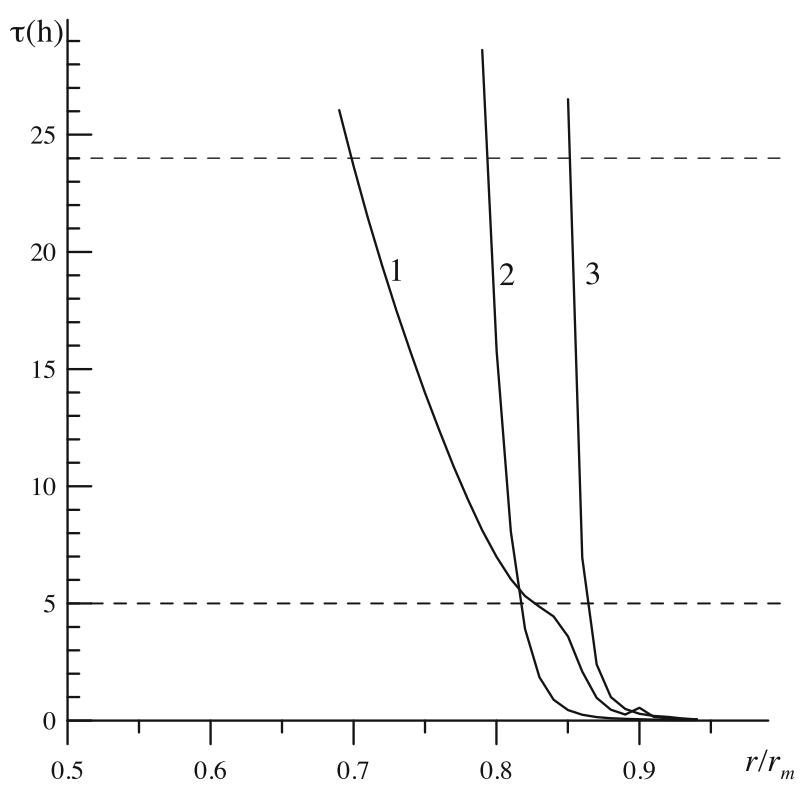

Fig. 7. Distribution over the radius of the characteristic time $\tau$ needed for the asymptotic regime of the magnetospheric convection to set in for different solar wind velocities in the magnetosheath. Curves 1,2 , 3 correspond to $v_{0}=200,400,800 \mathrm{~km} / \mathrm{s}$ for an average amplitude of stochastic FMS oscillations in the magnetosheath $\left\langle\left|B_{r}\right|\right\rangle \sim 1 \mathrm{nT}$.

It is in this range of magnetic shells that a maximum concentration of resonance surfaces for SMS waves is reached in our model geotail.

The obtained values of $\tau$ can be regarded as the upper limit of the time needed for the asymptotic regime to set in in the plasma flow. Time $\tau$ decreases quadratically when the amplitude of turbulent plasma oscillations in the magnetosheath increases. Moreover, a more accurate approach to solving the initial problem (19) must take into account contribution from MHD oscillations related to the evolution of a Kelvin-Helmholtz instability at the magnetopause. The solar wind being opaque for such oscillations (Leonovich, 2011a, b), the problem would be formulated in a different manner than in this work.

These oscillations do not provide a significant contribution to the oscillation amplitude in the solar wind far from the plasmapause. They can, however, produce a significant additional contribution to the oscillation amplitude in the magnetotail, in the region adjacent to the magnetopause. To determine this contribution it is necessary to specify the amplitude of these oscillations and solve the problem of determining their spatial structure. In contrast to oscillations in the magnetosheath, the amplitude of these oscillations is uncertain, varying strongly as per the solar wind parameters. Therefore, to avoid unnecessary complications of the problem, the Kelvin-Helmholtz instability-related oscillations were not taken into account in the above suggested approach. If the flux of unstable waves in the geotail is assumed to be comparable with the wave flux discussed in this work, we may expect a 1.5-2 fold increase in the amplitude of the resonant SMS waves. As follows from the above calculations, this means a 2-3-fold decrease in the characteristic time $\tau$ as well as a somewhat wider range of magnetic shells on which the asymptotic regime of magnetospheric 


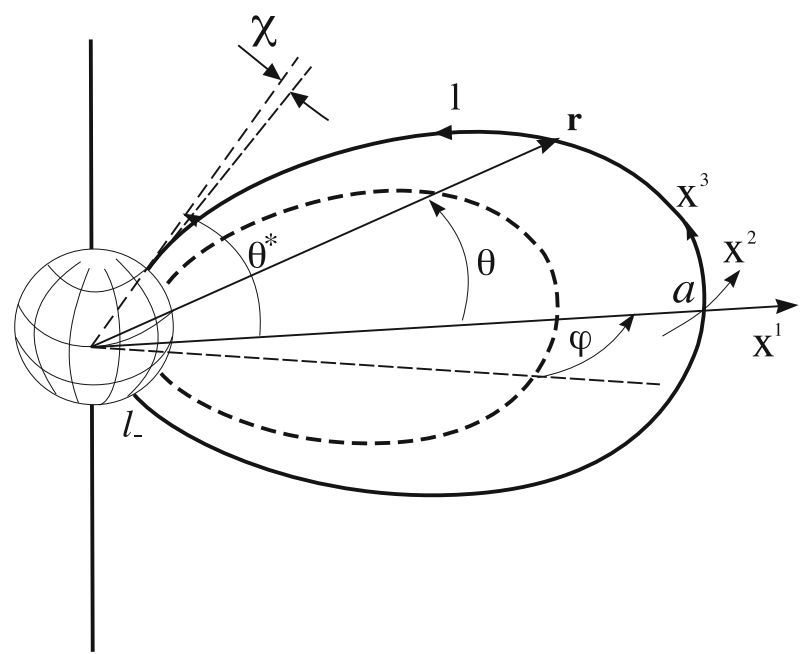

Fig. 8. The curvilinear orthogonal coordinate system $\left(x^{1}, x^{2}, x^{3}\right)$ of the dipole-like magnetic field lines and the non-orthogonal coordinate system $(a, \phi, \theta)$ used in numerical calculations.

convection can set in.

\section{Magnetosonic Resonance in a 2-D Inhomoge- neous Dipole-Like Magnetosphere}

Let us now address the features of magnetosonic resonance in a dipole-like magnetosphere (Fig. 8). Unlike the media models discussed above, resonance in this case arises on closed field lines. The result is that, due to the boundary conditions imposed on the ionosphere, SMS oscillations form standing waves along field lines.

Let us introduce a curvilinear orthogonal coordinate system $\left(x^{1}, x^{2}, x^{3}\right)$, in which the coordinate $x^{3}$ is along the field line, $x^{1}$ is across the magnetic shells, and the azimuthal $x^{2}$ coordinate completes the right hand coordinate system. The squared length element in this coordinate system is found as

$$
d s^{2}=g_{1}\left(d x^{1}\right)^{2}+g_{2}\left(d x^{2}\right)^{2}+g_{3}\left(d x^{3}\right)^{2},
$$

where $g_{i}(i=1,2,3)$ are metric coefficients. We assume that the plasma and magnetic field are homogeneous along the azimuthal coordinate $x^{2}$.

It is convenient to describe the MHD field components via electromagnetic potentials. According to the Helmholtz expansion theorem (Korn and Korn, 1968), an arbitrary vector field, at any point of which its first derivative is determined, can be represented as a sum of the potential and vortex fields. For the two-dimensional vector $\mathbf{E}=$ $\left(E_{1}, E_{2}, 0\right)$ this expansion has the form

$$
\mathbf{E}=-\nabla_{\perp} \varphi+\left[\nabla_{\perp}, \Psi\right]
$$

where $\nabla_{\perp} \equiv\left(\nabla_{1}, \nabla_{2}\right)$ is the transverse 2 -dimensional gradient, $\varphi$ and $\Psi$ are the scalar and vector potentials, respectively. Under proper gauge calibration, the vector potential has a longitudinal (field-aligned) component only, $\Psi=\left(0,0, \psi_{3} \equiv \psi\right)$. Using the linearized system (1)(4) we express the perturbed magnetic field components through the potentials $\varphi$ and $\psi$ as

$$
\begin{aligned}
& B_{1}=\frac{c}{\omega} \frac{g_{1}}{\sqrt{g}} \nabla_{3}\left(k_{2} \varphi-i \frac{g_{2}}{\sqrt{g}} \nabla_{1} \psi\right), \\
& B_{2}=\frac{c}{\omega} \frac{g_{2}}{\sqrt{g}} \nabla_{3}\left(i \nabla_{1} \varphi+k_{2} \frac{g_{1}}{\sqrt{g}} \psi\right), \\
& B_{3}=i \frac{c}{\omega} \frac{g_{3}}{\sqrt{g}}\left(\nabla_{1} \frac{g_{2}}{\sqrt{g}} \nabla_{1} \psi-k_{2}^{2} \frac{g_{1}}{\sqrt{g}} \psi\right) .
\end{aligned}
$$

After some transformation, the system of linearized equations (1)-(4) is reduced to a system of two related equations for the potentials $\varphi$ and $\psi$ (see Leonovich et al., 2006)

$$
\begin{gathered}
\nabla_{1} \widehat{L}_{T} \nabla_{1} \varphi \\
-k_{2}^{2}\left(\widehat{L}_{P} \varphi+\frac{S^{2}}{A^{2}} \frac{\varphi}{\sqrt{g_{1} g_{2}}} \nabla_{1} \ln B_{0} \nabla_{1} \ln \frac{\sqrt{g_{3}} P_{0}^{\sigma}}{B_{0}}\right) \\
=i k_{2}\left(\nabla_{1} \widehat{L}_{T} \frac{g_{1}}{\sqrt{g}} \psi-\widehat{L}_{P} \frac{g_{2}}{\sqrt{g}} \nabla_{1} \psi\right) \\
\frac{B_{0} \sqrt{g_{3}}}{4 \pi \rho_{0}} \widehat{L}_{0} \frac{B_{0}}{\sqrt{g_{3}}} \widetilde{\Delta} \psi+S^{2} \bar{\Delta} \psi+\omega^{2} \psi \\
=-i \frac{B_{0} \sqrt{g_{3}}}{4 \pi k_{2} \rho_{0}} \widehat{L}_{0} B_{0} \widehat{L}_{T} \nabla_{1} \varphi \\
-i \varphi k_{2} S^{2} \frac{g_{3}}{\sqrt{g}} \nabla_{1} \ln \frac{\sqrt{g_{3}} P_{0}^{\sigma}}{B_{0}} .
\end{gathered}
$$

where $p=\sqrt{g_{2} / g_{1}}, \sigma=1 / \gamma$. In (26) we introduced the toroidal $\widehat{L}_{T}$ and poloidal $\widehat{L}_{P}$ longitudinal operators, as follows

$$
\begin{aligned}
& \widehat{L}_{T}=\frac{1}{\sqrt{g_{3}}} \nabla_{3} \frac{p}{\sqrt{g_{3}}} \nabla_{3}+p \frac{\omega^{2}}{A^{2}} \\
& \widehat{L}_{P}=\frac{1}{\sqrt{g_{3}}} \nabla_{3} \frac{p^{-1}}{\sqrt{g_{3}}} \nabla_{3}+p^{-1} \frac{\omega^{2}}{A^{2}}
\end{aligned}
$$

and in (27) the longitudinal operator $\widehat{L}_{0}$

$$
\widehat{L}_{0}=\frac{\gamma}{\omega^{2}} \frac{P_{0}^{1-\sigma}}{\sqrt{g}} \nabla_{3} \frac{\sqrt{g}}{g_{3}} \frac{P_{0}^{\sigma}}{\rho_{0}} \nabla_{3}+1,
$$

and the differential operators analogous to the Laplace operator:

$$
\begin{array}{r}
\widetilde{\Delta}=\frac{g_{3}}{\sqrt{g}} \nabla_{1} \frac{g_{2}}{\sqrt{g}} \nabla_{1}-\frac{k_{2}^{2}}{g_{2}}+\nabla_{3} \frac{g_{2}}{\sqrt{g}} \nabla_{3} \frac{g_{1}}{\sqrt{g}}, \\
\bar{\Delta}=\frac{B_{0}}{P_{0}^{\sigma}} \frac{1}{\sqrt{g_{1} g_{2}}}\left(\nabla_{1} \frac{p P_{0}^{\sigma}}{B_{0}} \nabla_{1}-\frac{k_{2}^{2}}{p} \frac{P_{0}^{\sigma}}{B_{0}}\right. \\
\left.+\nabla_{3} \frac{\sqrt{g}}{g_{3}} \frac{P_{0}^{\sigma}}{\rho_{0}} \nabla_{3} \frac{\rho_{0}}{B_{0} \sqrt{g_{3}}}\right) .
\end{array}
$$

In a homogeneous plasma, the right-hand parts of these equations vanish. The operator in the left-hand part of (26) then provides the dispersion equation for the Alfven waves $\omega^{2}=k_{\|}^{2} A^{2}$, where $k_{\|}^{2} \equiv k_{3}^{2} / g_{3}$ is the field-aligned component of the wave vector. The operator in the lefthand part of (27) yields the dispersion equation for the slow and fast magnetosonic waves:

$$
\omega^{4}-\omega^{2} k^{2}\left(A^{2}+S^{2}\right)+k^{2} k_{\|}^{2} A^{2} S^{2}=0,
$$


where $k^{2}=k_{\|}^{2}+k_{\perp}^{2}$ is the squared total wave vector, and $k_{\perp}^{2}=k_{1}^{2} / g_{1}+k_{2}^{2} / g_{2}$ is the squared transverse wave vector component. Thus, Alfven oscillations are described by the scalar potential $\varphi$, and magnetosonic modes are characterized by the longitudinal component $\psi$ of the vector potential. The solution of the dispersion equation (28) can be represented as

$$
\omega^{2}=\frac{k^{2}}{2}\left(A^{2}+S^{2}\right) \pm \sqrt{\frac{k^{4}}{4}\left(A^{2}+S^{2}\right)^{2}-k^{2} k_{\|}^{2} A^{2} S^{2}}
$$

Here the plus/minus sign corresponds to the FMS/SMS waves. If one of the inequalities $S \ll A, A \ll S$, or $\left|k_{\|}\right| \ll\left|k_{\perp}\right|$ holds, the following approximate dispersion equations can be obtained: $\omega^{2} \approx k^{2} C_{F}^{2}$ for the FMS waves, where $C_{F}^{2}=A^{2}+S^{2}$ and $\omega^{2} \approx k_{\|}^{2} C_{S}^{2}$ for the SMS waves, where $C_{S}^{2}=A^{2} S^{2} /\left(A^{2}+S^{2}\right)$.

In an inhomogeneous plasma, the right-hand parts of (26) and (27) describe the interaction of the Alfven and magnetosonic modes. Although the potential $\psi$ describes both the fast and slow magnetosonic modes, in the linear approximation this potential can be decomposed as the sum of the component $\psi_{F}$, related to the FMS wave, and $\psi_{S}$, related to the SMS wave, that is $\psi=\psi_{F}+\psi_{S}$. Away from the resonance surface, the main contribution to potential $\psi$ comes almost exclusively from the FMS oscillations $\left(\psi \approx \psi_{F}\right)$. Neglecting the small component $(\sim S / A \ll 1)$ related to the derivatives along the longitudinal coordinate $x^{3}$ in the operator $\widehat{L}_{0}$ in (27), we obtain an equation that describes the FMS wave field far from the resonant surface:

$$
A^{2} \widetilde{\Delta} \psi_{F}+S^{2} \bar{\Delta} \psi_{F}+\omega^{2} \psi_{F}=0
$$

An approximate solution of (29) was found in Leonovich and Mazur $(2000,2001)$ where feedback from the mode driven by the FMS wave was shown to be small, thus making it possible to use the decoupled equation (29) to describe FMS oscillations throughout the entire region of their existence, even inside the resonant region.

For the magnetosphere the typical eigenfrequencies of fundamental harmonics of standing Alfven and SMS waves, as determined by the background plasma, differ considerably. This means that interaction between the Alfven and SMS waves, possible in a finite-pressure plasma embedded in a curved magnetic field (Southwood and Saunders, 1985), is negligible. While examining the SMS oscillation structure described by Eq. (27) one may put $\varphi=0$ in its right-hand part. Therefore, in the vicinity of the resonant surface we obtain the equation for the resonant SMS oscillations:

$$
\begin{aligned}
& \frac{B_{0} \sqrt{g_{3}}}{4 \pi \rho_{0}} \widehat{L}_{0} \frac{B_{0}}{\sqrt{g_{3}}} \widetilde{\Delta} \psi_{S}+S^{2} \bar{\Delta} \psi_{S}+\omega^{2} \psi_{S} \\
& =\frac{A^{2} S^{2}}{\omega^{2}} \frac{\rho_{0} / \sqrt{g_{1} g_{2}}}{B_{0} P_{0}^{\sigma}} \nabla_{3} \frac{\sqrt{g}}{g_{3}} \frac{P_{0}^{\sigma}}{\rho_{0}} \nabla_{3} \frac{B_{0}}{\sqrt{g_{3}}} \widetilde{\Delta} \psi_{F} .
\end{aligned}
$$

The right-hand part of (30) represents the drivermonochromatic FMS wave field - that will be treated as a function known from the solution of (29). At the frequencies in question, the magnetosphere as a whole is an opacity region for FMS. If we assume the source of FMS oscillations to be either outside, or at the boundary of, the magnetosphere, their amplitude decreases exponentially inside the magnetosphere on a scale proportional to $m$. FMS oscillations with $m \gg 1$ practically do not penetrate into the magnetosphere. Only oscillations with $m \sim 1$ on resonant shells have an amplitude sufficient to drive SMS waves effectively. Therefore, we shall consider oscillations with $m \sim 1$.

The boundary condition for SMS waves on the ionosphere, taking into account its finite conductivity, has the form (Leonovich and Mazur, 1996; Leonovich et al., 2006)

$$
\left.\psi_{S}\right|_{l=l_{ \pm}}=\left.\mp i \frac{v_{ \pm}}{\omega} \frac{\partial \psi_{S}}{\partial l}\right|_{\ell=\ell_{ \pm}},
$$

where the signs " \pm " refer to the intersection points of the field lines with the Northern and Southern ionospheres; $\ell$ is the coordinate measured along the field line from the equator, $d \ell=\sqrt{g_{3}} d x^{3}$,

$$
v_{ \pm}=\frac{c^{2} \cos \chi_{ \pm}}{4 \pi \Sigma_{p}^{( \pm)}}
$$

$\Sigma_{p}^{( \pm)}$is the height-integrated Pedersen conductivity of the ionosphere.

As we will see, the typical scale of resonant SMS oscillations across magnetic shells is much smaller than their longitudinal wave length, $\left|\nabla_{1} \psi_{s} / \psi_{s}\right| \gg\left|\nabla_{3} \psi_{s} / \psi_{s}\right|$. Therefore, a solution to (30) may be sought using the method of different scales, representing the potential $\psi_{S}$ as

$$
\psi_{S}=U\left(x^{1}\right)\left(S\left(x^{1}, x^{3}\right)+h\left(x^{1}, x^{3}\right)\right)
$$

where the function $U\left(x^{1}\right)$ describes, in the main order, the small-scale transverse structure of oscillations along the $x^{1}$ coordinate, whereas the function $S\left(x^{1}, x^{3}\right)$ describes the oscillation structure along magnetic field lines. The typical scale of $S\left(x^{1}, x^{3}\right)$ along $x^{1}$ is assumed to be much larger than the scale of $U\left(x^{1}\right)$. The small correction term $h\left(x^{1}, x^{3}\right)$ describes the oscillation structure in higher orders of the perturbation theory.

An equation for the longitudinal structure can be obtained if one retains in (30) only the main-order terms $\left(\sim \nabla_{1}^{2} \psi_{S}\right)$ of perturbation theory:

$$
\frac{\partial}{\partial \ell} \alpha\left(x^{1}, \ell\right) \frac{\partial H}{\partial \ell}+\frac{\omega^{2}}{C_{S}^{2}} \alpha\left(x^{1}, \ell\right) H=0,
$$

where $H\left(x^{1}, x^{3}\right)=S\left(x^{1}, x^{3}\right) B_{0} /\left(g_{2} \sqrt{g_{3}}\right), \alpha\left(x^{1}, \ell\right)=$ $P_{0}^{\sigma} \sqrt{g_{1} g_{2}} / \rho_{0}$. We assume that, in the main order, the functions $S\left(x^{1}, x^{3}\right)$ satisfy the homogeneous boundary conditions in the ionosphere: $S\left(x^{1}, x_{ \pm}^{3}\right)=0$. The solution of (33), with such boundary conditions, is a series of eigenfunctions $S_{N}\left(x^{1}, \ell\right)$ and corresponding eigenfrequencies $\Omega_{S N}\left(x^{1}\right)$, where $N=1,2,3 \ldots$ is the longitudinal wavenumber. In the two first orders of the WKB approximation, the solution of (33) satisfying the above boundary conditions has the form

$$
S_{N}\left(x^{1}, x^{3}\right)=\sqrt{\frac{2}{t_{S}} \frac{g_{3} g_{1}^{3 / 2}}{P_{0}^{\sigma} g_{2}^{1 / 2}} \frac{C_{S}}{A^{2}}} \sin \left(\Omega_{S N} \int_{l_{-}}^{l} \frac{d l}{C_{S}}\right) .
$$



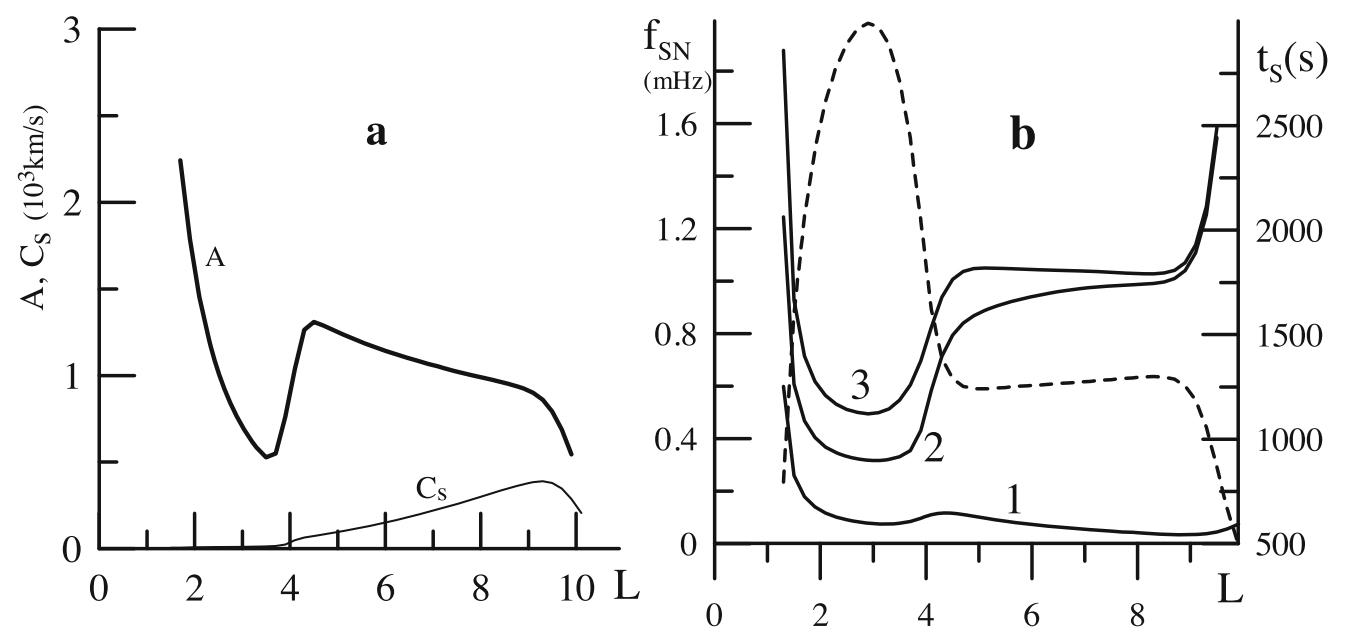

Fig. 9. (a) Equatorial distribution of the Alfven speed $A$ and the SMS waves speed $C_{S}$ across the magnetic shells $\left(L=a / R_{E}, R_{E}\right.$ is Earth radius). (b) Distribution across the magnetic shells of eigenfrequencies $f_{S N}=\Omega_{S N} / 2 \pi$ of the first three harmonics of standing SMS waves and the travel time along the field line between conjugate ionospheres $t_{S}$ at the SMS wave velocity $C_{S}$.
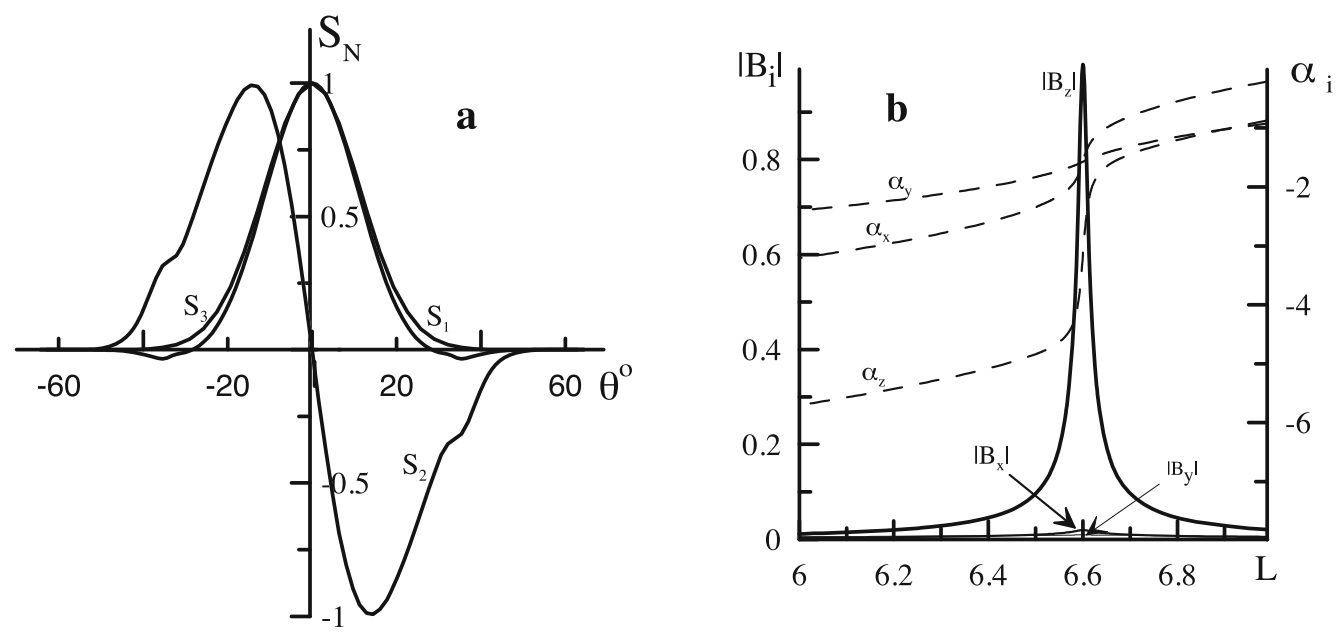

Fig. 10. The spatial structure of the resonant SMS oscillations. (a) Structure along the magnetic field lines of the first three harmonics of standing SMS waves $S_{N}(\theta)(N=1,2,3)$ at the magnetic shell $a=6.6 R_{E}$. (b) Transverse structure of magnetic field components of the first harmonic $(N=1)$ : the amplitude $\left|B_{i}\right|$ and phase $\alpha_{i}$ distributions close to the equatorial plane $(i=x, y, z)$.

where $\Omega_{S N}=\pi N / t_{s}$,

$$
t_{s}=\int_{\ell_{-}}^{\ell_{+}} \frac{d \ell^{\prime}}{C_{s}}
$$

is the travel time along a field line between the magnetoconjugate ionospheres at SMS wave speed. The eigenfunctions (34) are normalized by the following condition

$$
\int_{l_{-}}^{l_{+}} \frac{p P_{0}^{\sigma}}{g_{1} g_{3}} \frac{A^{2}}{C_{S}^{2}} S_{N}^{2} d l=1
$$

Only numerical solutions can be found to equations (33), however, describing the longitudinal structure of the fundamental and low- $N$ magnetosonic harmonics. For a numerical solution, we use the coordinate system $(a, \phi, \theta)$ related to the dipole magnetic field lines (see Fig. 8). The plasma distribution is set using a self-consistent model of the dipole magnetosphere (Leonovich et al., 2004). The radial distributions of the Alfven and magnetosonic speeds in the equatorial magnetospheric plane derived from this model are shown in Fig. 9(a). Such a distribution of plasma parameters is typical of the Earth's dayside magnetosphere.

All the following calculations concern the magnetic shell corresponding to the geosynchronous orbit, $a=6.6 R_{E}$. Figure 9(b) shows the radial distributions of the eigenfrequencies of the first three harmonics of standing SMS waves, obtained from a numerical solution of (33) for the ionosphere under homogeneous boundary conditions. The same figure displays the distribution of transit time $t_{S}$ determining, in the WKB approximation, the frequencies of standing SMS waves. It is easily verifiable that the numerically calculated frequencies of the first harmonics differ significantly from the WKB ones. They occupy the lowestfrequency part of the spectrum of MHD oscillations observed in Earth's magnetosphere $(f \lesssim 1 \mathrm{mHz})$.

Figure 10(a) shows the field-aligned structures of the first three harmonics of standing SMS waves. The fundamental harmonics of standing SMS waves differ from their WKB representation (34) radically. The main peculiarity of fundamental SMS harmonics is a rapid decrease in ampli- 
tude when approaching the ionosphere. Such a structure of $S_{N}\left(x^{1}, x^{3}\right)$ results in a number of important consequences. First, resonant SMS oscillations are impossible to detect on the ground or by a low-orbit satellite. Second, the ionosphere cannot be an absorber of the resonant SMS wave energy. SMS wave damping in the magnetosphere is caused by their resonant interaction with the background plasma particles.

Note that such a structure of standing SMS waves is only typical of long magnetic field lines in the outer magnetosphere. In the inner plasmasphere (on magnetic shells $L<$ 2 ), the distribution of standing SMS wave amplitudes on short field lines is such as to feature sharp peaks at the ionospheric $F_{2}$-region altitudes (Leonovich et al., 2010). Moreover, the plasma ion to electron temperature ratio in this plasmaspheric region is such that SMS waves exhibit weak enough dissipation $\left(T_{e}>2 T_{i}\right.$ and $\left.\gamma / \omega \sim 10\right)$. Therefore, standing SMS waves can exist in the plasmasphere long enough, thus making their registration possible-based, for example, on observations of the ionospheric total electron concentration variations as detected by the GPS network receivers (Afraimovich et al., 2009).

Let us now address the structure of resonant SMS oscillations across magnetic shells. Let us pre-multiply (30) by $S_{N} p P_{0}^{\sigma} / \sqrt{g_{3}} S^{2}$ and integrate along the field line between the conjugate ionospheres. The correction term $h_{N}\left(x^{1}, x^{3}\right)$ in (32) satisfies the following ionospheric boundary condition (see (31))

$$
\left.h_{N}\right|_{l=l_{ \pm}}=\left.\mp i U_{N}\left(x^{1}\right) \frac{v_{ \pm}}{\omega} \frac{\partial S_{N}}{\partial l}\right|_{l=l_{ \pm}} .
$$

Given this boundary condition and (33), we obtain the following equation for function $U_{N}\left(x^{1}\right)$ :

$$
\begin{aligned}
& \frac{\left(\omega+i \gamma_{N}\right)^{2}-\Omega_{S N}^{2}}{\omega^{2}} \nabla_{1}^{2} U_{N}^{2} \\
& \quad-\left[\beta_{1 N}+\left(k_{2}^{2} \beta_{2 N}+\beta_{3 N}\right) \frac{\left(\omega+i \gamma_{N}\right)^{2}-\Omega_{S N}^{2}}{\omega^{2}}\right] U_{N} \\
& =\Gamma_{N}
\end{aligned}
$$

where

$$
\begin{aligned}
& \beta_{1 N}=\int_{l_{-}}^{l_{+}} \frac{p P_{0}^{\sigma}}{g_{3}} S_{N}\left(\nabla_{3} \frac{g_{2}}{\sqrt{g}} \nabla_{3} \frac{g_{1}}{\sqrt{g}} S_{N}+\frac{\Omega_{S N}^{2}}{C_{S}^{2}} S_{N}\right) d l \\
& \beta_{2 N}=\int_{l_{-}}^{l_{+}} \frac{p P_{0}^{\sigma}}{g_{2} g_{3}} \frac{A^{2}}{C_{S}^{2}} S_{N}^{2} d l \\
& \beta_{3 N}=-\int_{l_{-}}^{l_{+}} \frac{p P_{0}^{\sigma}}{g_{3}} \frac{A^{2}}{S^{2}} S_{N} \nabla_{3} \frac{g_{2}}{\sqrt{g}} \nabla_{3} \frac{g_{1}}{\sqrt{g}} S_{N} d l \\
& \Gamma_{N}=\frac{\Omega_{S N}^{2}}{\omega^{2}} \int_{l_{-}}^{l_{+}} \frac{p P_{0}^{\sigma}}{g_{3}} \frac{A^{2}}{C_{S}^{2}} S_{N} \widetilde{\Delta} \psi_{F} d l .
\end{aligned}
$$

Here, the damping decrement, $\gamma_{N}$, for each of the harmonics of standing SMS waves is determined, near the reso- nance surface, by the plasma ion to electron temperature ratio.

Let the function $\Omega_{S N}\left(x^{1}\right)$ change monotonically, so that a linear dependence

$$
\Omega_{S N}\left(x^{1}\right) \approx \omega\left(1-\frac{x^{1}-x_{S N}^{1}}{L}\right)
$$

be used to approximate $\Omega_{S N}\left(x^{1}\right)$ in the vicinity of the resonant surface.

This approximation is valid at $\left|x^{1}-x_{S N}^{1}\right| \ll L$, where $L=\left|\partial \ln \Omega_{S N} / \partial x^{1}\right|^{-1}$ is the typical scale of the $\Omega_{S N}$ variation at $x^{1}=x_{S N}^{1}$. Substituting (38) into (37) and introducing the dimensionless variable $\xi=\left(x^{1}-x_{S N}^{1}\right) / \lambda_{S N}$, where $\lambda_{S N}=1 / \sqrt{\beta_{3 N}}$, we obtain an equation describing the transverse structure of magnetosonic resonance

$$
(\xi+i \varepsilon) \frac{\partial^{2} U_{N}}{\partial \xi^{2}}-\left[c_{N}+\left(1+d_{N}\right)(\xi+i \varepsilon)\right] U_{N}=G_{N}
$$

The coefficients of this equation are: $\varepsilon=\gamma_{N} L / \omega \lambda_{S N}$ is the dimensionless width of the resonance, $c_{N}=\beta_{1 N} L \lambda_{S N}$, $d_{N}=\beta_{2 N} k_{2}^{2} \lambda_{S N}^{2}$, and $G_{N}=\Gamma_{N} \lambda_{S N} L$. These coefficients may be considered as constants because they vary insignificantly within the localization region of the desired solution $U_{N}(\xi)$.

Solution to (39) has the form (see Leonovich and Mazur, 1997)

$$
U_{N}(\xi)=i G_{N}(0) \int_{0}^{\infty} \frac{\exp [i k(\xi+i \varepsilon)+i \zeta(k)]}{k^{2}+1+d_{N}} d k
$$

where

$$
\zeta(k)=\frac{c_{N}}{\sqrt{1+d_{N}}} \arctan \frac{k}{\sqrt{1+d_{N}}} .
$$

As $\xi \rightarrow 0$ the bulk of the integrand (40) accumulates in the domain $k \gg 1$, making it possible to set $\zeta(k) \approx \zeta(\infty)$ in the exponent, while neglecting all the terms but $k^{2}$ in the denominator. This yields

$$
U_{N}(\xi) \stackrel{\xi \rightarrow 0}{\approx} G_{N}(0) e^{i \zeta(\infty)}(\xi+i \varepsilon) \ln (\xi+i \varepsilon) .
$$

In the asymptotic $|\xi| \rightarrow \infty$ the bulk of the integrand (40) accumulates in the domain $k \ll 1$, making it possible to set $k=0$ in $\zeta(k)$ and in the denominator. The integral is then easily calculated

$$
U_{N}(\xi) \stackrel{|\xi| \rightarrow \infty}{\approx}-\frac{G_{N}(0)}{1+d_{N}} \frac{1}{\xi+i \varepsilon}
$$

Thus, the amplitude of the resonant SMS oscillations away from the resonant plane decreases asymptotically as $\propto$ $|\xi|^{-1}$. This behavior satisfies the boundary conditions on the $x^{1}$ coordinate-resonant oscillations have a finite amplitude far from the resonance surface. The magnetic field components of the oscillation near the resonance surface are 
described by following expressions

$$
\begin{aligned}
& B_{1 N} \approx-i \frac{\bar{B}_{N}}{\lambda_{S N}} \ln (\xi+i \varepsilon)\left(\frac{g_{1}}{\sqrt{g}} \nabla_{3} \frac{g_{2}}{\sqrt{g}} S_{N}\right), \\
& B_{2 N} \approx k_{2} \bar{B}_{N}(\xi+i \varepsilon) \ln (\xi+i \varepsilon)\left(\frac{g_{2}}{\sqrt{g}} \nabla_{3} \frac{g_{1}}{\sqrt{g}} S_{N}\right), \\
& B_{3 N} \approx i \frac{\bar{B}_{N}}{\lambda_{S N}^{2}} \frac{1}{(\xi+i \varepsilon)} \frac{S_{N}}{g_{1}}
\end{aligned}
$$

where $\bar{B}_{N}=(c / \omega) G_{N}(0) e^{i \zeta(\infty)}$. The longitudinal (compressional) magnetic component $B_{3 N}$ has the strongest singularity, $\propto \xi^{-1}$. The radial magnetic component $B_{1 N}$ has a weaker logarithmic singularity, and the azimuthal component $B_{2 N}$ is regular. Figure 10(b) shows the radial amplitude-phase structure of the physical components of the wave magnetic field $\left(B_{x} \equiv B_{1} / \sqrt{g_{1}}=\left|B_{x}\right| e^{i \alpha_{x}}, B_{y} \equiv\right.$ $B_{2} / \sqrt{g_{2}}=\left|B_{y}\right| e^{i \alpha_{y}}$, and $\left.B_{z} \equiv B_{3} / \sqrt{g_{3}}=\left|B_{z}\right| e^{i \alpha_{z}}\right)$ of the fundamental harmonic $(N=1)$ of SMS waves near the resonant magnetic shell $a=6.6 R_{E}$. The response to the FMS wave is normalized in such a manner as to make the peak value $\left|B_{z}\right|=1$ at the resonance surface.

The initial oscillation phase is chosen to be zero in an asymptotically distant region right of the resonant shell. For numerical calculations the damping rate and the imaginary correction factor were chosen to be rather small, $\varepsilon=10^{-2}$, to expose the resonant structure. The amplitude of the resonant SMS oscillations is controlled by the FMS wave amplitude and the SMS damping rate. When $\gamma_{N}$ and $\varepsilon$ increase, the maximum amplitude decreases and the resonant peak widens. Passing through the resonant peak the phase of the compressional $B_{z}$ component changes approximately by $\pi$, the phase of the $B_{x}$ component by $\sim \pi / 2$, while the phase of the $B_{y}$ component remains practically the same.

\section{Conclusion}

In this paper we would like to emphasize the possibility of resonant conversion of large-scale fast magnetosonic waves into localized slow magnetosonic oscillations. Several magnetospheric processes are discussed in which magnetosonic resonance may play an appreciable role.

1. The problem of the magnetosonic waves incidence on and reflexion from the plasma transition layer is solved. The conditions for the Alfven and magnetosonic resonances are realized when FMS waves pass from the solar wind into the magnetosphere through the magnetopause, as well as in the regions of well-developed ring current in the magnetosphere. Some field components of resonant oscillations have singularities on the resonance surfaces, in the absence of dissipation.

To regularize singularities near the resonant surfaces, effective decrements are introduced both for the Alfven and SMS waves as imaginary additions to the oscillation frequency. Dissipation of the Alfven oscillations is small enough. The decrement of the SMS oscillations, in contrast, can be rather large. Its value is determined by the plasma ion to electron temperature ratio near the resonant surface for the SMS wave. Oscillations reaching the resonant surface for SMS waves are absorbed completely in its neighbourhood.
It was shown that, if dissipation of oscillations near the resonant surfaces for SMS waves is large enough, the presence of strongly decaying resonant SMS oscillations in the system changes the wave field substantially. When $\gamma_{s} \sim \omega$ this influence extends up to the resonant surface for the Alfven waves. Specifically, the hodograph rotation direction is not reversed for monochromatic oscillations when we pass through the Alfven resonant surface. This phenomenon is applicable for identifying the presence of a resonance surface for SMS waves in the plasma configuration under consideration.

2. Magnetosonic resonance may be used to transfer the momentum from fast magnetosonic waves penetrating into the geotail lobes from the magnetosheath, to the background plasma. A rather wide (in frequency and wave numbers) range of waves is shown to exist for which the conditions for magnetosonic resonance are satisfied in the geotail. The highest concentration of resonance shells is achieved in the geotail regions adjacent to the magnetopause. The framework of quasilinear theory is used to obtain an approximate solution to the equation describing the evolution of the plasma ion distribution function under the impact of an MHD wave flux. It is shown that, on the time asymptotic (when $t \rightarrow \infty$ ), an Earthward plasma flow moving at 50$150 \mathrm{~km} / \mathrm{s}$ is established in the geotail lobe regions adjoining the magnetopause.

The characteristic time $\tau$ needed for the asymptotic regime to set in in the plasma flow in the regions adjoining the magnetopause was found to be comparable with the mean time interval $\sim 3-6 \mathrm{~h}$, during which the geotail configuration can be regarded as stable. It increased sharply on the inner magnetic shells, however. Thus, the FMS wave flux moving from the magnetosheath into the magnetopause transfers its momentum to plasma ions in the geotail lobes which is capable of forming an Earthward flow of magnetospheric convection. This mechanism may explain the formation of an Earthward flow of magnetospheric convection in the geotail lobes (on open field lines) during prolonged periods of the Northern IMF component.

3. The problem of the spatial distribution of resonant SMS oscillation field in an axi-symmetric magnetosphere with a dipole-like magnetic field is solved. These oscillations are peculiar in that their longitudinal structure represents a wave standing along field lines due to boundary conditions on the ends of field lines crossing the highly conductive ionosphere twice. On the long field lines examined in this work (at the $L \sim 5-10$ magnetic shells) the oscillation amplitude is maximum near the equatorial surface decreasing rapidly away from it. Therefore, resonant SMS oscillations can only be observed near the magnetospheric equatorial plane.

The frequency spectra of the fundamental harmonics of standing Alfven and SMS waves differ by about two orders of magnitude, so that an effective coupling between these two branches of MHD oscillations is impossible in a mildly disturbed magnetosphere. If dissipation of SMS waves is not too high-allowing their resonant structure to be conspicuous enough - it is the longitudinal components of the magnetic field and velocity of the oscillations that have the largest amplitude. Passing through the resonant peak, the 
phase of the compressional component $B_{\|}$changes by $\pi$.

Acknowledgments. This work was partially supported by Program of presidium of Russian Academy of Sciences \#22 and grants 12-02-0031-a, 13-05-90436-Ukr_f_a from the Russian Foundation for Basic Research.

\section{Appendix A. Local Decrement of SMS Waves}

Let us consider a homogeneous plasma the parameters of which are equal to the parameters at the point of magnetosonic resonance, $x=x_{S}$, under consideration. The dispersion equation for low-frequency oscillations of plasma with a maxwellian distribution of particles over velocities (see Akhiezer et al., 1974) is

$$
1+\sum_{\alpha=i, e} \frac{\omega_{p \alpha}^{2}}{k^{2} v_{\alpha}^{2}}\left[1+i \sqrt{\pi} z_{0}^{\alpha} e^{x_{\alpha}} \sum_{n=-\infty}^{\infty} I_{n}\left(x_{\alpha}\right) w\left(z_{n}^{\alpha}\right)\right]=0
$$

where summation is with respect to the kind of the particles (the $\alpha$ index denotes the plasma ions and electrons) and to the cyclotron harmonics (the $n$ index). The notation is: $k$ is the wave vector module, $x_{\alpha}=k_{\perp}^{2} \rho_{\alpha}^{2}, \rho_{\alpha}=v_{\alpha} / \omega_{\alpha}$ is the Larmor radius, $\omega_{\alpha}=e B_{0} / m_{\alpha} c$ is the cyclotron frequency, $\omega_{p \alpha}=\sqrt{4 \pi n_{\alpha} e^{2} / m_{\alpha}}$ is the plasma frequency and $v_{\alpha}=\sqrt{T_{\alpha} / m_{\alpha}}$ is the thermal velocity of particles of the $\alpha$ kind, $z_{n}^{\alpha}=\left(\omega-n \omega_{\alpha}\right) / \sqrt{2} k_{z} v_{\alpha}$. The modified Bessel function $I_{n}\left(x_{\alpha}\right)$ is approximately represented as $I_{n}\left(x_{\alpha}\right) \approx\left(x_{\alpha} / 2\right)^{n} / n$ ! when the values of the argument are small (we will assume the condition $\left|k_{\perp} \rho_{\alpha}\right| \ll 1$ to be satisfied). Function $w(z)$ is the probability integral having the following asymptotic representations (see Abramowitz and Stegun, 1964):

$$
\begin{aligned}
w(z) & =e^{-z^{2}}\left(1+\frac{2 i}{\sqrt{\pi}} \int_{0}^{z} e^{t^{2}} d t\right) \\
& \approx \begin{cases}1-z^{2}+2 i z / \sqrt{\pi}, & |z| \ll 1, \\
\exp \left(-z^{2}\right)+i / \sqrt{\pi} z, & |z| \gg 1\end{cases}
\end{aligned}
$$

In the known extreme case (see Akhiezer et al., 1974) $v_{i} \ll$ $\left|\omega / k_{z}\right| \ll v_{e}$, it is possible to confine ourselves to the zero harmonics in the sum with respect to $n$ in (A.1) and write down the dispersion equation approximately as

$$
\frac{\omega_{p i}^{2}}{k^{2}}\left(\frac{1}{v_{s}^{2}}\left(1+i \sqrt{\pi} z_{e}^{0}\right)-\frac{k_{z}^{2}}{\omega^{2}}\right) \approx 0
$$

where $\omega_{p i}^{2} / v_{s}^{2}=\omega_{p e}^{2} / v_{e}^{2}, v_{s}=\sqrt{T_{e} / m_{i}}$. In the zero-order perturbation theory the solution of this equation gives, for the extreme case $T_{e} \gg T_{i}$, the dispersion equation for SMS waves: $\omega^{2}=k_{z}^{2} v_{s}^{2}$. Taking into account the next order of perturbation theory, we obtain the dispersion equation including the oscillation energy absorption

$$
\omega^{2}=k_{z}^{2} v_{s}^{2}\left(1-i \sqrt{\frac{\pi m_{e}}{2 m_{i}}}\right) .
$$

In this extreme case $\left(T_{e} / T_{i} \rightarrow \infty\right)$ the value of interest is $\bar{\varepsilon}_{s}=\bar{\gamma}_{s} / \operatorname{Re}(\omega) \equiv \bar{\varepsilon}_{s \infty}=-\sqrt{\pi m_{e} / 2 m_{i}} / 2 \approx-0.015$. The total solution of (A.1) calculated numerically in the range $10^{-2}<T_{e} / T_{i}<10^{2}$ is presented in Fig. A.1. The calculated curve $\bar{\varepsilon}_{s}\left(T_{e} / T_{i}\right)$ has a universal form in a wide

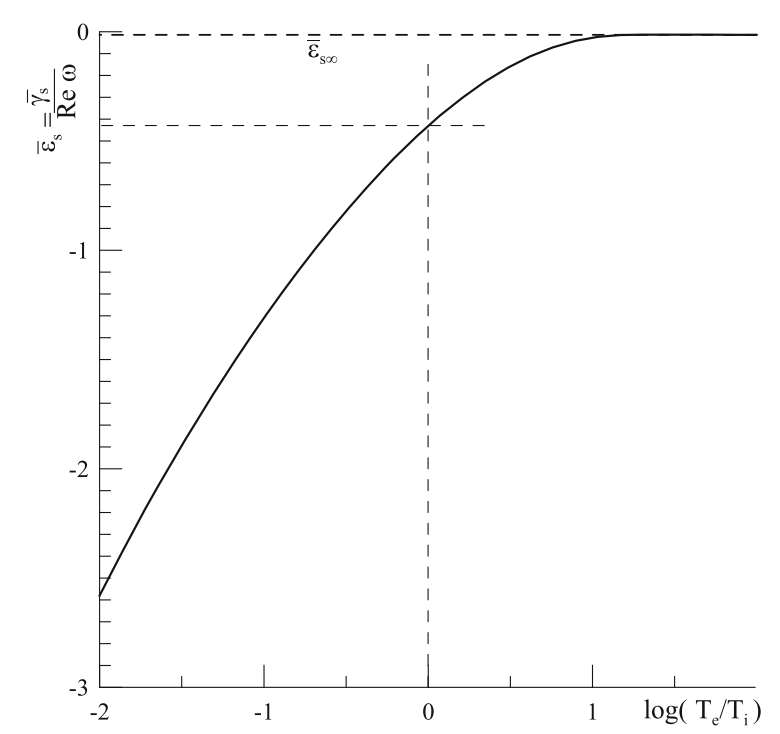

Fig. A.1. SMS oscillation decrement $\bar{\gamma}_{s} \equiv \operatorname{Im} \omega$ to frequency $\operatorname{Re} \omega$ ratio versus the plasma non-isothermality parameter $\log \left(T_{e} / T_{i}\right)$.

enough range of variation of the plasma parameters ( $1 \mathrm{nT} \leq$ $\left.B_{0} \leq 10 \mathrm{~T} ; 1 \mathrm{~km} / \mathrm{s} \leq A \leq 10^{4} \mathrm{~km} / \mathrm{s} ; 10^{-2} \leq \beta \leq 1\right)$.

It should be noted, however, that, unlike the Alfven oscillations, the damping decrement of SMS oscillations for $T_{e} / T_{i} \geq 1$ is rather large $\left(\left|\bar{\varepsilon}_{s}\right| \sim 1\right)$. Therefore the regularizing factor $\varepsilon_{s}$ in the denominator of (10), should be localized near $x=x_{S}$ on such a scale where the oscillations in question can be treated as SMS waves. Obviously, this scale is determined by the size of the transparency region for the SMS waves $\Delta_{s}=x_{s}-x_{01}$. If the linear expansion for the Alfven speed of the form $A^{2}(x) \approx$ $A_{S}^{2}\left[1-\left(x-x_{S}\right) / a_{s}\right]$ is applicable near $x_{S}, x_{01}$, we have $\Delta_{s} \approx k_{z}^{2} a_{s} \beta^{*} /\left[\left(k_{z}^{2}+k_{y}^{2}\right)\left(1+\beta^{*}\right)^{2}-2 k_{z}^{2} \beta^{*}\right]$. For small magnitudes $\beta^{*} \ll 1$ this scale $\Delta_{s} \approx a_{s} \beta^{*} k_{z}^{2} /\left(k_{z}^{2}+k_{y}^{2}\right)$ is much smaller than the scale $a_{s}=\left(\partial \ln \left(A^{2}(x)\right) / \partial x\right)_{x=x_{s}}^{-1}$, and when $\beta^{*} \sim 1$ they are comparable. To localize the decrement of SMS oscillations near $x=x_{S}$ we will perform a substitution $\omega \rightarrow \omega-i \gamma_{s}$ in the denominator of (10), where the model expression

$$
\gamma_{s}=-\bar{\gamma}_{s} \exp \left[-\left(x-x_{s}\right)^{2} / \Delta_{s}^{2}\right]
$$

will be chosen for the decrement $\gamma_{s}$, or, similarly, $\varepsilon_{s}=$ $-\bar{\varepsilon}_{s} \exp \left(-\left(x-x_{s}\right)^{2} / \Delta_{s}^{2}\right)$ used in the numerical calculations. Obviously, this approach cannot be used for the case of SMS waves incident on the transition layer. These waves will decay strongly in their entire region of existence.

\section{Appendix B. Ion Distribution Function as Trans- formed under the Impact of Resonant SMS Waves}

In the presence of SMS waves, the asymptotic form of the equation for the ion distribution function (when $t \rightarrow \infty$ ) has the form (see Sizonenko and Stepanov, 1968; Akhiezer et al., 1974)

$$
\frac{\partial f}{\partial t} \approx \frac{\partial}{\partial v_{\|}} D \frac{\partial f}{\partial v_{\|}}
$$


where

$$
\begin{aligned}
D= & \pi\left(\frac{e}{m_{i}}\right)^{2} \int d^{3} \mathbf{k}\left|E_{3} J_{0}(\lambda)+i \frac{k_{\|} v_{\perp}}{\omega} J_{0}^{\prime}(\lambda) E_{2}\right|^{2} \\
& \times \delta\left(\omega-k_{\|} v_{\|}\right)
\end{aligned}
$$

is the ion diffusion coefficient in the velocity space. Here, $f \equiv f\left(v_{\|}, v_{\perp}, t\right)$ is the ion distribution function over velocities, $v_{\|, \perp}$ are ion velocities along and across magnetic field lines, $J_{0}(\lambda)$ is the Bessel function, $\lambda=k_{\perp} v_{\perp} / \omega_{i}, k_{\|}, \mathbf{k}_{\perp}$ are the parallel and perpendicular wave vector components, $\omega_{i}$ is ion gyrofrequency, and the various components of field oscillations are subscripted with $i=1,2,3: E_{3} \equiv\left\langle E_{\|}\right\rangle$is the averaged amplitude of the oscillation electric field along the magnetic field after averaging over the wave number spectrum, $E_{1}$ is the average amplitude of the field along $\mathbf{k}_{\perp}$, and $E_{2}$ is the average amplitude along the vector $\left[\mathbf{B}_{\mathbf{0}} \mathbf{k}_{\perp}\right]$. The relationship between the oscillation frequency and the wave vector components is determined by the local dispersion equation.

In the ideal MHD approximation, $E_{3}=0$ for all types of MHD waves, while for the $E_{2}$ component we have

$$
E_{2}=\left\langle-\frac{\omega}{k_{z} c} \frac{k_{r} \tilde{B}_{r}+k_{\phi} \tilde{B}_{\phi}}{k_{\perp}}\right\rangle,
$$

the angular brackets \langle\rangle denote averaging over the wave vector phases, where $k_{r}, k_{\phi}, k_{z}$ are the radial, azimuthal and parallel components of the local Cartesian coordinate system in a cylindrical model, and the tilde above denotes a spatial harmonic of the Fourier transform. For example

$$
\tilde{B}_{r}=\frac{1}{(2 \pi)^{3 / 2}} \int d^{3} \mathbf{r} B_{r}(\mathbf{r}) e^{-i \mathbf{k r}} .
$$

Note that $E_{2}=0$ for the Alfven waves, while the $B_{r}$ component of the SMS wave field is singular, and the $B_{\phi}$ component has a finite amplitude in the absence of dissipation on the resonance surface. Using the natural assumption that the ion Larmor radius $\rho_{i}$ is small as compared to the wavelength $k_{\perp} \rho_{i}=k_{\perp} v_{T i} / \omega_{i} \ll 1$, where $v_{T i} \sim v_{\perp}$ is ion thermal velocity, we obtain

$$
D \approx \frac{\pi}{4} \frac{v_{\perp}^{4}}{B_{0}^{2}} \int d^{3} \mathbf{k}\left\langle\left|k_{r} \tilde{B}_{r}\right|^{2}\right\rangle \delta\left(\omega-k_{z} v_{\|}\right)
$$

for the diffusion coefficient.

From the solution of (17) we know the structure of the wave field harmonic of the form

$$
\begin{aligned}
\bar{B}_{r}(r, & \left.m, k_{z}, \omega\right) \\
\quad= & \frac{1}{(2 \pi)^{3 / 2}} \int B_{r}(r, t) e^{-i\left(k_{z} z+m \phi-\omega t\right)} d \phi d z d t,
\end{aligned}
$$

for which the diffusion coefficient can be written as

$$
D \approx \frac{\pi}{4} \frac{v_{\perp}^{4}}{v_{\|} B_{0}^{2}} \sum_{m=0}^{\infty} \int_{0}^{\infty}\left\langle\left|\nabla_{r} \bar{B}_{r}\left(r, m, k_{z}=\omega / v_{\|}, \omega\right)\right|^{2}\right\rangle d \omega .
$$

Here the integrand $\delta$ function was used for integrating over $k_{z}$. Averaging is over the phases of the frequency harmonic, as well as over the azimuthal and parallel harmonics of the wave vector. Thus it is clear that coupling occurs when the parallel wave phase velocity $\omega / k_{\|}$is the same as the parallel plasma ion velocity $v_{\|}$.

As the initial condition for solving (B.1), we use the Maxwell distribution function

$$
f\left(v_{\|}, v_{\perp}\right)=\frac{n_{0}}{\pi^{3 / 2} v_{T i}^{3}} \exp \left(-\frac{v_{\|}^{2}+v_{\perp}^{2}}{v_{T i}^{2}}\right) .
$$

This function describes the equilibrium plasma state in the absence of waves.

As follows from the form of the diffusion coefficient (B.2), Eq. (B.1) does not change the dependence of the distribution function on $v_{\perp}$. Integrating (B.1) over $v_{\perp}$, we obtain

$$
\frac{\partial \bar{f}}{\partial t} \approx \frac{\partial}{\partial v_{\|}} \bar{D} \frac{\partial \bar{f}}{\partial v_{\|}}
$$

where

$$
\begin{aligned}
\bar{f}\left(v_{\|}\right) & =\int_{0}^{2 \pi} d \phi \int_{0}^{\infty} v_{\perp} f\left(v_{\|}, v_{\perp}\right) d v_{\perp} \\
& =\frac{n_{0}}{\sqrt{\pi} v_{T i}} \exp \left(-\frac{v_{\|}^{2}}{v_{T i}^{2}}\right),
\end{aligned}
$$

$$
\begin{aligned}
\bar{D} & =\frac{1}{\pi v_{T i}^{2}} \int_{0}^{2 \pi} d \phi \int_{0}^{\infty} v_{\perp} D e^{-v_{\perp}^{2} / v_{T i}^{2}} d v_{\perp} \\
& \approx \frac{\pi}{2} \frac{v_{T i}^{4}}{v_{\|} B_{0}^{2}} \sum_{m=0}^{\infty} \int_{0}^{\infty}\left\langle\left|\nabla_{r} \bar{B}_{r}\left(r, m, k_{z}=\omega / v_{\|}, \omega\right)\right|^{2}\right\rangle d \omega .
\end{aligned}
$$

\section{References}

Abramowitz, M. and I. A. Stegun (editors), Handbook of Mathematical Functions, National Bureau of Standards Applied Mathematics Series 55, 832 pp., USA, 1964.

Afraimovich, E. L., I. K. Edemskiy, A. S. Leonovich, L. A. Leonovich, S. V. Voeykov, and Y. V. Yasyukevich, MHD nature of night-time MSTIDs excited by the solar terminator, Geophys. Res. Lett., 36, L15106, doi:10.1029/2009GL039803, 2009.

Agapitov, O., K.-H. Glassmeier, F. Plaschke, H.-U. Auster, D. Constantinescu, V. Angelopoulos, W. Magnes, R. Nakamura, C. W. Carlson, S. Frey, and J. P. McFadden, Surface waves and field line resonances: A THEMIS case study, J. Geophys. Res., 114, A00C27, doi:10.1029/2008JA013553, 2009.

Akhiezer, A. I., I. A. Akhiezer, R. V. Polovin, A. G. Sitenko, and K. N. Stepanov, Plasma Electrodynamics, Nauka, Moscow, 237 pp., 1974 (in Russian).

Borovsky, J. E., M. F. Thomsen, R. C. Elphic, T. E. Cayton, and D. J. McComac, The transport of plasma sheet material from the distant tail to geosynchronous orbit, J. Geophys. Res., 103, 20297-20331, 1998.

Chen, L. and A. Hasegawa, A theory of long period magnetic pulsation. 1. Steady state excitation of field line resonances, J. Geophys. Res., 79, 1024-1032, 1974.

Cheng, C. Z. and C. S. Lin, Eigenmode analysis of compressional waves in the magnetosphere, Geophys. Res. Lett., 14, 884-887, 1987.

Cheng, C.-C., J.-K. Chao, and T.-S. Hsu, Evidence of the coupling of a fast magnetospheric cavity mode to field line resonances, Earth Planets Space, 50, 683-697, 1998.

Cheremnykh, O. K., A. S. Parnowski, and O. S. Burdo, Ballooning modes in the inner magnetosphere of the Earth, Planet. Space Sci., 52, 12171229, 2004.

Goldstein, M. L., D. A. Roberts, and W. H. Matthaeus, Magnetohydrodynamic turbulence in the solar wind, Ann. Rev. Astron. Astrophys., 33, $283-325,1995$ 
Gonzalez, A. G. and J. Gratton, Magnetoacoustic surface gravity waves, Sol. Phys., 134, 211-232, 1991.

Inhester, B., Numerical modelling of hydromagnetic wave coupling in the magnetosphere, J. Geophys. Res., 92, 4751-4756, 1987.

Klimushkin, D. Yu., Theory of azimuthally small-scale hydromagnetic waves in the axisymmetric magnetosphere with finite plasma pressure, Ann. Geophys., 16, 303-321, 1998.

Klimushkin, D. Yu. and P. N. Mager, On the spatial structure and dispersion of slow magnetosonic modes coupled with Alfven modes in planetary magnetospheres due to field line curvature, Planet. Space Sci., 56, 1273-1279, 2008

Korn, G. A. and T. M. Korn, Mathematical Handbook for Scientists and Engineers, McGraw-Hill Book Company, 1968.

Lee, D.-H. and R. L. Lysak, Monochromatic ULF wave coupling in the dipole model: The impulsive excitation, J. Geophys. Res., 94, 1709717109,1989

Leonovich, A. S., MHD-instability of the magnetotail: Global modes, Planet. Space Sci., 59, 402-411, 2011a.

Leonovich, A. S., A theory of MHD instability of an inhomogeneous plasma jet, J. Plasma Phys., 77, 315-337, $2011 \mathrm{~b}$

Leonovich, A. S. and D. A. Kozlov, Alfvénic and magnetosonic resonances in a nonisothermal plasma, Plasma Phys. Control. Fus., 51, 085007, doi:10.1088/0741-3335/51/8/085007, 2009.

Leonovich, A. S. and V. A. Mazur, Resonance excitation of standing Alfvén waves in an axisymmetric magnetosphere (monochromatic oscillations), Planet. Space Sci., 37, 1095-1108, 1989.

Leonovich, A. S. and V. A. Mazur, Penetration to the Earth's surface of standing Alfvén waves excited by external currents in the ionosphere, Ann. Geophys., 14, 545-556, 1996.

Leonovich, A. S. and V. A. Mazur, A model equation for monochromatic standing Alfvén waves in the axially symmetric magnetosphere, J. Geophys. Res., 102, 11443-11456, 1997.

Leonovich, A. S. and V. V. Mishin, An energy flux of magnetosonic waves from the solar wind into magnetosphere, Geomagnetizm $i$ aeronomiya, 39, 52-58, 1999 (in Russian)

Leonovich, A. S. and V. A. Mazur, Structure of magnetosonic eigenoscillations of an axisymmetric magnetosphere, J. Geophys. Res., 105, 2770727716, 2000.

Leonovich, A. S. and V. A. Mazur, On the spectrum of magnetosonic eigenoscillations of an axisymmetric magnetosphere, J. Geophys. Res., 106, 3919-3928, 2001.

Leonovich, A. S., V. V. Mishin, and J. B. Cao, Penetration of magnetosonic waves into the magnetosphere: Influence of a transition layer, Ann. Geophys., 21, 1083-1093, 2003.

Leonovich, A. S., V. A. Mazur, and J. B. Cao, Self-consistent model of a dipole-like magnetosphere with an azimuthal solar wind flow, J. Plasma Phys., 70, 99-111, 2004.

Leonovich, A. S., D. A. Kozlov, and V. A. Pilipenko, Magnetosonic resonance in a dipole-like magnetosphere, Ann. Geophys., 24, 2277-2289, 2006

Leonovich, A. S., D. A. Kozlov, and I. K. Edemskiy, Standing slow magnetosonic waves in a dipole-like plasmasphere, Planet. Space Sci., 58, 1425-1433, 2010

Marsh, E. and C.-Y. Tu, Spectral and spatial evolution of compressive turbulence in the inner solar wind, J. Geophys. Res., 95, 11945-11956, 1990.

Matthaeus, W. H. and M. L. Goldstein, Measurement of the rugged invariants of magnetohydrodynamics turbulence in the solar wind, J. Geophys. Res., 87, 6011-6028, 1982.

Mazur, V. A. and A. S. Leonovich, ULF hydromagnetic oscillations with the discrete spectrum as eigenmodes of MHD-resonator in the nearEarth part of the plasma sheet, Ann. Geophys., 24, 1639-1648, 2006.

Miles, A. J. and B. Roberts, On the properties of magnetoacoustic surface waves, Sol. Phys., 119, 257-278, doi:10.1007/BF00146179, 1989.

Nakariakov, V. M., L. Ofman, E. E. DeLuca, B. Roberts, and J. M. Davila, TRACE observation of damped coronal loop oscillations: Implications for coronal heating, Science, 285, 862, doi:10.1126/science.285.5429.862, 1999.

Ohtani, S., A. Miura, and T. Tamao, Coupling between Alfven and slow magnetosonic waves in an inhomogeneous finite- $\beta$ plasma-I. Coupled equations and physical mechanism, Planet. Space Sci., 37, 567-577, 1989.

Pilipenko, V. A., ULF waves on the ground and in space, J. Atmos. Terr Phys., 52, 1193-1209, 1990.

Radoski, H. R., A theory of latitude dependent geomagnetic micropulsations: The asymptotic fields, J. Geophys. Res., 79, 595-604, 1974.

Rankin, R., K. Kabin, J. Y. Lu, I. R. Mann, R. Marchand, I. J. Rae, V. T. Tikhonchuk, and E. F. Donovan, Magnetospheric field-line resonances: Ground-based observations and modeling, J. Geophys. Res., 110, A10S09, doi:10.1029/2004JA010919, 2005.

Rankin, R., K. Kabin, and R. Merchand, Alfvénic field line resonances in arbitrary magnetic field topology, Adv. Space Res., 38, 1720-1729, 2006.

Sergeev, V. A. and N. A. Tsyganenko, The Earth's Magnetosphere, Moscow, Nauka, 1980 (in Russian).

Sizonenko, V. L. and K. N. Stepanov, Quasilinear theory of plasma oscillations with linear dispersion, Ukrainian J. Phys., 13, 876-878, 1968.

Southwood, D. J., Some features of field line resonances in the magnetosphere, Planet. Space Sci., 22, 483-492, 1974.

Southwood, D. J. and M. A. Saunders, Curvature coupling of slow and Alfvén MHD waves in a magnetotail field configuration, Planet. Space Sci., 33, 127-134, 1985.

Tamao, T., Transmission and coupling resonance of hydromagnetic disturbances in the non-uniform Earth's magnetosphere, Sci. Rep. Tohoku Univ., 5, 17, 43-54, 1965.

Titheridge, J. E., Temperatures in the upper ionosphere and plasmasphere, J. Geophys. Res., 103, 2261-2277, 1998.

Walker, A. D. M., Theory of magnetospheric standing hydromagnetic waves with large azimuthal wave number. 1 . Coupled magnetosonic and Alfvén waves, J. Geophys. Res., 92, 10039-10045, 1987.

Yumoto, K., Characteristics of localized resonance coupling oscillations of the slow magnetosonic wave in a non-uniform plasma, Planet. Space Sci., 33, 1029-1036, 1985.

A. S. Leonovich (e-mail: leon@iszf.irk.ru) and D. A. Kozlov 